$10 / 3.94856$

SANDIA REPORT

SAND92-1706 - UC-906

Unlimited Release

Printed January 1994

\title{
Blind Deconvolution of Two-Dimensional Complex Data
}

D. C. Ghiglia, L. A. Romero

\section{Prepared by}

Sandla National Leboratories

Albuquerque, Now Moxico 87485 and Livermore, Callfornle 94560

for thu Unitod 8tates Dopertment of Enorgy

undor Contract DE-ACO4-04AL85000 
Issued by Sandia National Laboratories, operated for the United States Department of Energy by Sandia Corporation.

NOTICE: This report was prepared as an account of work sponsored by an agency of the United States Government. Neither the United States Government nor any agency thereof, nor any of their employees, nor any of their contractors, subcontractors, or their employees, makes any warranty, express or implied, or assumes any legal liability or responsibility for the accuracy, completeness, or usefulriess of any information, apparatus, product, or process disclosed, or represents that its use would not infringe privately owned rights. Reference herein to any specific commercial product, process, or service by trade name, trademark, manufacturer, or otherwise, does not necessarily constitute or imply its endorsement, recommendation, or favoring by the United States Government, any agency thereof or any of their contractors or subcontractors. The views and opinions expressed herein do not necessarily state or reflect those of the United States Government, any agency thereof or any of their contractors.

Printed in the United States of America. This report has been reproduced directly from the best available copy.

Available to DOE and DOE contractors from Office of Scientific and Technical Information

PO Box 62

Oak Ridge, TN 37831

Prices available from (615) 576-8401, FTS 626-8401

Available to the public from

National Technical Information Service

US Department of Commerce

5285 Port Royal Rd

Springfield, VA 22161

NTIS price codes

Printed copy: A03

Microfiche copy: A01 
SAND92-1706

Unlimited Release

Printed January 1994

Distribution

Category UC-906

\title{
Blind Deconvolution of Two-Dimensional Complex Data
}

\author{
D. C. Ghiglia \\ Algorithms and Discrete Mathematics Deparment \\ L. A. Romero \\ Applied and Numerical Mathematics Department \\ Sandia National Laboratories \\ Albuquerque, NM 87185
}

\begin{abstract}
Inspired by the work of Lane and Bates on automatic multidimensional deconvolution [J. Opt. Soc. Am. A 4, 180 (1987)], we have developed a systematic approach and an operational code for performing the deconvolution of multiply-convolved two-dimensional complex data sets in the absence of noise. We explain, in some detail, the major algorithmic steps, where noise or numerical errors can cause problems, our approach in dealing with numerical rounding errors, and where special noise-mitigating techniques can be used toward making blind deconvolution practical. Several examples of deconvolved imagery are presented, and future research directions are noted.
\end{abstract}

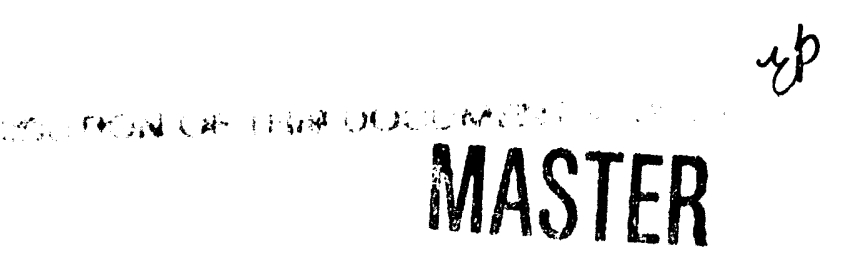


Intentionally Left Blank 


\section{Contents}

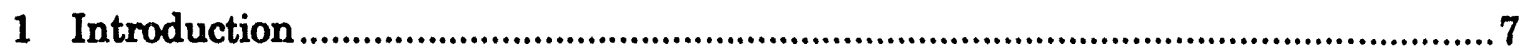

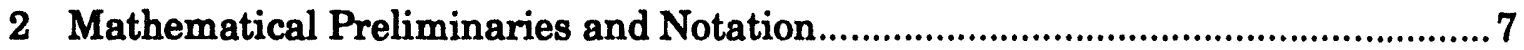

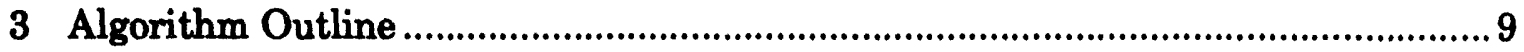

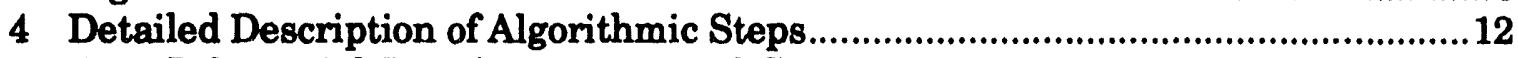

4.1 Polynomial Root Association and Grouping ............................................12

4.2 Root Tracking and Fourier-Transform Factor Generation …......................20

4.3 Transform Matrix Equalization and Deconvolved Image Formation ...........23

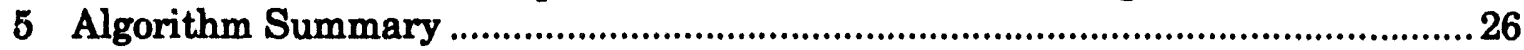

6 Examples of Deconvolved Complex Imagery ………..........................................2

7 Summary and Conclusion.................................................................................

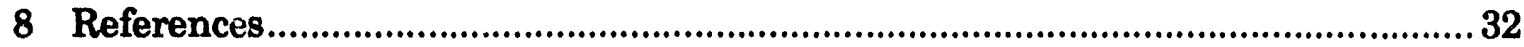

\section{Figures}

1 Typical rendition of $u$ space and $v$ space for a complex quadratic polynomial .....12

2 Typical root distribution resulting from specific $u$................................................13

3 Typical $u$ path causing roots $v_{i}$ and $v_{j}$ to coverage at some point $v_{0} \ldots \ldots \ldots \ldots \ldots \ldots \ldots \ldots . .17$

4 Typical root grouping .................................................................................................. 19

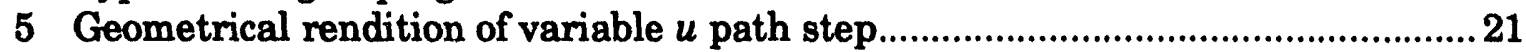

6 Blind deconvolution example ..................................................................................28

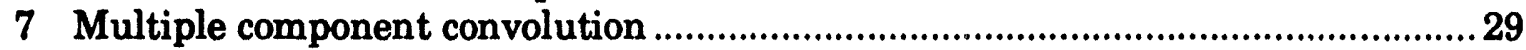

8 Multiple component convolution ……...................................................................30

9 Multiple component convolution ............................................................................... 31 


\section{Intentionally Left Blank}




\section{Blind Deconvolution of Two-Dimensional Complex Data}

\section{Introduction}

Linear systems theory indicates that the output $g(x)$ of a linear shift-invariant system is equal to the input $f(x)$ convolved with the system response function $h(x)$. Mathematically,

$$
g(x)=\int f(x-\tau) h(\tau) d \tau,
$$

where the integral and the variables $\mathrm{x}$ and $\tau$ can be considered as multidimensional if necessary.

Any linear system imparts its own type of distortion or signature on the input. Since no system is perfect, it is important to be able to recover or to estimate the input adequately by somehow removing the distorting effects of the system.

Conventional deconvolution is the process by which the input is estimated by using knowledge of the system response and the measured output. Noise, uncertainties, and model errors make conventional deconvolution problems difficult to solve. Even with noiseless data and perfect knowledge, deconvolution is not trivial.

It seems reasonable to assume that deconvolution without knowledge of the degrading system response, or point-spread function (i.e., blind deconvolution), is theoretically impossible. Surprising as it may sound, however, blind deconvolution has been shown to be possible in two or more dimensions (e.g., images), whereas it is theoretically impossible with one-dimensional (1-D) signals. ${ }^{1}$

Inspired by the work of Lane and Bates ${ }^{1}$ and a perverse sense of pleasure in seemingly being able to fool Mother Nature, we sought to develop a systematic method backed by robust numerical methods that would permit two-dimensional (2-D) blind deconvolution without reliance on visualization of zero sheets, manual intervention to involve their separation, or special constraints. ${ }^{2 \cdot 5}$

Thus far we have developed a systematic approach and an operational code for performing the deconvolution of multiple 2-D complex data sets in the absence of noise. We will explain, in some detail, the major algorithmic steps, where noise or numerical errors can cause problems, our approach in dealing with numerical rounding errors, and where special noise-mitigating techniques can be used toward making blind deconvolution practical.

\section{Mathematical Preliminaries and Notation}

From Fourier-transform theory, the $k$-fold convolution of functions can be represented in the transform domain as the $k$-fold product of the individual transforms. Mathematically,

$$
f_{1}(x, y) \bullet f_{2}(x, y) \bullet \ldots \bullet f_{k}(x, y) \Leftrightarrow F_{1}\left(\omega_{x}, \omega_{y}\right) F_{2}\left(\omega_{x}, \omega_{y}\right) \ldots F_{k}\left(\omega_{x}, \omega_{y}\right),
$$


where the centered dots denote 2-D convolution and the double arrow denotes forward and inverse Fourier transformation.

In the transform domain, the output, $F\left(\omega_{x}, \omega_{y}\right)$, of a cascade of $k$ linear systemseach with transfer function $F_{l}\left(\omega_{x}, \omega_{y}\right)$ is

$$
F\left(\omega_{x}, \omega_{y}\right)=\prod_{l=1}^{k} F_{l}\left(\omega_{x}, \omega_{y}\right) .
$$

When one is dealing with sampled data, the continuous Fourier transform is replaced with a discrete Fourier transform (DFT) with no loss of generality. For 2-D functions this discrete DFT can be written as a polynomial in the complex variables $u$ and $v$ (as is shown below). Evaluation of this polynomial for values of $u$ and $v$ at equally spaced points around the unit circle yields the DFT.

Thus, if $f(m, n)$ represents the multiply-convolved image (of compact support), the 2-D DFT can be written as

$$
F(k, l)=\sum_{n=0}^{N-1} \sum_{m=0}^{M-1} f(m, n) \exp [-j(2 \pi k m / M)] \times \exp [-j(2 \pi \ln / N)]
$$

The finite-degree 2-D polynomial representing the transform is obtained with a simple change of variables and is written as

$$
F(u, v)=\sum_{n=0}^{N-1 M-1} \sum_{m=0}^{M} f(m, n) u^{m} v^{n}
$$

where $u=\exp (-j 2 \pi k / M)$ and $v=\exp (-j 2 \pi l / N)$.

The correct factoring of this 2-D polynomial [as in Eq. (3)] yields all the transform components (up to an arbitrary complex scale factor and possible inconsequential linear phase terms ${ }^{6-8}$ ) corresponding to all the convolution components. The lack of the fundamental theorem of algebra for polynomials of two or more dimensions indicates that multidimensional polynomials are not generically factorizable. If a 2-D polynomial is factorizable, that factorization is almost always unique. Therefore, blind deconvolution of 2-D functions is equivalent to factoring a 2-D polynomial.

Our systematic approach to blind deconvolution is based on factoring the 2-D polynomial (zero-sheet separation) by manipulating the 1-D polynomial that is obtained from Eq. (5) by writing complex coefficients based on $f(m, n)$ and $u$. From Eq. (5) it is easy to see that

$$
F(u, v)=\sum_{n=0}^{N-1} a_{n}(u) v^{n}
$$

where

$$
a_{n}(u)=\sum_{m=0}^{M-1} f(m, n) u^{m}
$$


We now have a 1-D polynomial whose coefficients depend on the data $f(m, n)$ and the complex variable $u$. For any value of $u$ we can find the $M-1$ roots, $v_{i}(u), i=1,2, \ldots, M-1$ that depend on $u$. Since $u$ is a free parameter, it can be quite useful in our work.

We have partitioned the blind deconvolution algorithm into three major operations that we will highlight here and explain in more detail below. The first we call polynomial root association and grouping. Its function is to determine how many transform factors exist and which roots belong to which factor.

The second operation is root tracking and Fourier-transform factor generation. Its function is to track the roots as $u$ moves to each sequential Fourier point on the unit circle. Knowing which root belongs to which factor for a specific $u$ allows us to build up the transform matrices one column at a time. The first two operations are then repeated with the roles of $u$ and $v$ reversed or, equivalently, with the input data transposed so that a different set of transform matrices can be built up (effectively) one row at a time.

The final step is transform matrix equalization and deconvolved image formation. Its function is to pair up properly the transform matrices that are built up columnwise with its equivalent matrix built up rowwise. Once the transform matrices are properly paired up, the arbitrary row/column scalings (which result from polynomials being evaluated only from knowledge of its roots) are removed and the equalized matrices are inverse transformed to form the deconvolved image components. We will now provide an outline of the algorithm and then explain each operation in some detail.

\section{Algorithm Outline}

In this section we present the basic steps for performing blind deconvolution and ignore all the subtle numerical difficulties so that we may clarify the algorithm. For simplicity let us assume that only two images are convolved together; however, the algorithm can be extended to an arbitrary number of images.

As was explained in Section 2, the problem of deconvolving two images by using a DFT relation is equivalent to factoring a 2-D polynomial. Let us assume that the polynomia! can be written as

$$
F(u, v)=F_{1}(u, v) F_{2}(u, v) .
$$

We want to determine the factors $F_{1}(u, v)$ and $F_{2}(u, v)$ up to arbitrary multiplicative constants. We begin by selecting a particular $u=u_{0}$ and use standard methods to find all the roots $v_{i}\left(u_{0}\right)$ of the 1-D polynomial $F\left(u_{0}, v\right)$. We now seek to divide the roots into two groups $S_{1}$ and $S_{2}$ such that the roots in $S_{1}$ are all the roots of the polynomial $F_{1}\left(u_{0}, v\right)$ and the roots in $S_{2}$ are all the roots of the polynomial $F_{2}\left(u_{0}, v\right)$. The technique for doing this is the most important part of the algorithm. Once one has figured out how to accomplish this root-grouping step the rest of the algorithm is conceptually (although not practically) quite simple.

To divide the roots into these two sets we begin by arbitrarily assigning root $v_{1}\left(u_{0}\right)$ to the set $S_{1}$. This raises the question whether the root $v_{2}\left(u_{0}\right)$ is in $S_{1}$ or $S_{2}$. To answer this question we vary the path of $u$ in the complex plane and keep track of both of the roots $v_{1}(u)$ and $v_{2}(u)$. We select a path for the complex variable $u$ that causes these roots eventually to come together. In subsection 4.1 we describe how we determine this path, but here we assume that the roots can in fact be driven together. 
Suppose that the roots come together when $u=\tilde{u}$ and $v=\tilde{v}$. At this point the implicit function theorem requires that

$$
\frac{\partial}{\partial v} F(\tilde{u}, \tilde{v})=0
$$

Also note that

$$
\frac{\partial}{\partial u} F(\tilde{u}, \tilde{v})=F_{1}(\tilde{u}, \tilde{v}) \frac{\partial}{\partial u} F_{2}(\tilde{u}, \tilde{v})+F_{2}(\tilde{u}, \tilde{v}) \frac{\partial}{\partial u} F_{1}(\tilde{u}, \tilde{v})
$$

If one of the roots is a root of $F_{1}$ and the other is a root of $F_{2}$, it is clear from Eq. (9) that

$$
\frac{\partial}{\partial u} F(\tilde{u}, \tilde{v})=0
$$

If both roots are associated with $F_{1}$ or both are associated with $F_{2}$, there is no reason that Eq. (10) should hold.

We are not aware of an example in which both the derivatives $F_{u}$ and $F_{\nu}$ vanish at the point where two roots come together, yet the two roots are on the same zero sheet. We were not able to prove that the vanishing of both of these derivatives implies that the roots are on different zero sheets, but we can argue that this will be the case generically (in the measure theoretical sense). Certainly, if we have an arbitrary polynomial, it is not generic to have both of these derivatives vanish when two roots merge. If they did vanish we could add almost any arbitrarily small perturbation to the polynomial, and this would no longer be the case. Thus it follows that, for any arbitrary polynomial, we do not expect both of these derivatives to vanish when the roots merge. Note that it is indeed a special condition when the polynomial does factor. If we assume that this factorizable polynomial is the product of polynomials that are not special, then we do not expect to have both derivatives vanish when two roots on the same zero sheet merge.

This is the basic idea behind our algorithm. We drive roots together and check to see whether Eq. (10) holds. If it holds, then the roots are associated with different polynomials. If it does not hold, the roots are associated with the same polynomial. In practice we use a more sophisticated numerical test than this, but the test is based on this idea. By carrying out this process for all pairs of roots, we are able to determine the two sets $S_{1}$ and $S_{2}$.

Once we have determined the two sets $S_{1}$ and $S_{2}$, we perform the same process with the roles of $u$ and $v$ reversed. This gives us roots $u_{i}\left(v_{0}\right)$ that are divided up into two sets $T_{1}$ and $T_{2}$. The roots in $T_{1}$ are all the roots of $F_{1}\left(u, v_{0}\right)$ or all the roots of $F_{2}\left(u, v_{0}\right)$. We cannot yet say which one of those polynomials the roots in $T_{1}$ are in fact associated with at this stage in the algorithm. The roots in $T_{2}$ are all the roots of the remaining polynomial.

We are now left with the problem of determining $F_{1}(u, v)$ and $F_{2}(u, v)$ orice we know the roots $v_{i}\left(u_{0}\right)$ and $u_{i}\left(v_{0}\right)$ and the sets $S_{1}, S_{2}, T_{1}$, and $T_{2}$. First, note that to determine the functions (images) $f_{1}$ and $f_{2}$ we need only to evaluate the polynomials $F_{1}$ and $F_{2}$ when both $u$ and $v$ are at discrete points around the unit circle. Starting at $u_{0}$ we move $u$ around the unit circle, ensuring that we keep the roots in their two separate groups. This is in practice a very difficult numerical process, as will be dis- 
cussed below, but here we just assume that by taking small enough steps in $u$ we can tell easily which roots belong in each set.

For each value of $u$ we can write

$$
F_{1}(u, v)=A(u) Q_{1}(u, v)=A(u) \prod_{i}\left[v-v_{i}(u)\right], \quad v_{i} \in S_{1} .
$$

Here the function $A(u)$ must be included because knowing the roots $v_{i}\left(u_{0}\right)$ of $F_{1}(u, v)$ only determines the polynomial up to the multiplicative constant $A(u)$.

We also move $v$ around the unit circle, ensuring that we keep track of the roots as before. If we knew that the roots $u_{i}$ in $T_{1}$ were associated with the polynomial $F_{1}$, we could write

$$
F_{1}(u, v)=B(v) P_{1}(u, v)=B(v) \prod_{i}\left[u-u_{i}(v)\right], \quad u_{i} \in T_{1} .
$$

Since we are only determining the polynomials up to an arbitrary multiplicative constant, we can assume that $A\left(u_{1}\right)=1$. We can thus determine the function $B(v)$ by using

$$
Q_{1}\left(u_{1}, v\right)=B(v) P_{1}\left(u_{1}, v\right)
$$

Since both $Q_{1}$ and $P_{1}$ are known functions, $B(v)$ is easily determined, and so is the function $F_{1}$. In practice we use a more robust algorithm that takes advantage of the great redundancy that is available because we can choose $u_{1}$ to be any point on the unit circle.

We assumed that $T_{1}$ was the set associated with the polynomial $F_{1}$. To check if this is indeed the case, we can carry out the above process by fixing $u$ to be a different value than $u_{1}$. If $T_{1}$ is in fact associated with $F_{1}$, we should get the same function $B(v)$ up to a multiplicative constant regardless of the value of $u_{1}$ that we use. If $T_{1}$ is associated with the function $F_{2}$, this will not be the case. We carry out this process by assuming first that $T_{1}$ is associated with $F_{1}$ and then assuming that $T_{2}$ is associated with $F_{1}$. We determine which is correct by choosing all the discrete values around the unit circle and finding which set gives the same function $B(v)$, up to a multiplicative constant, for every value of $u_{1}$. We then determine the function $F_{2}$ by an identical process. This completes the general description of the basic algorithm. 


\section{Detailed Description of Algorithmic Steps}

\subsection{Polynomial Root Association and Grouping}

Let us consider two complex planes defined by the complex variables $u$ and $v$. The complex plane in which $u$ is free to move will be called $u$-space, and the complex plane in which the roots $v_{i}(u)$ reside will be called $v$ space. For a quadratic polynomial with complex coefficients $a_{n}(u)$, where $n=0,1,2, u$ space and $v$ space might resemble the schematics shown in Figure 1 for a particular value of $u=u_{0}$.

u-space

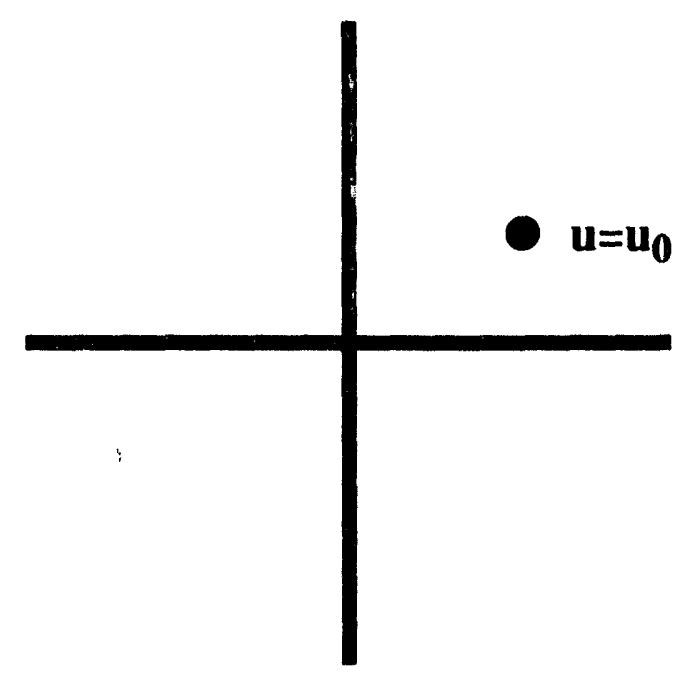

v-space

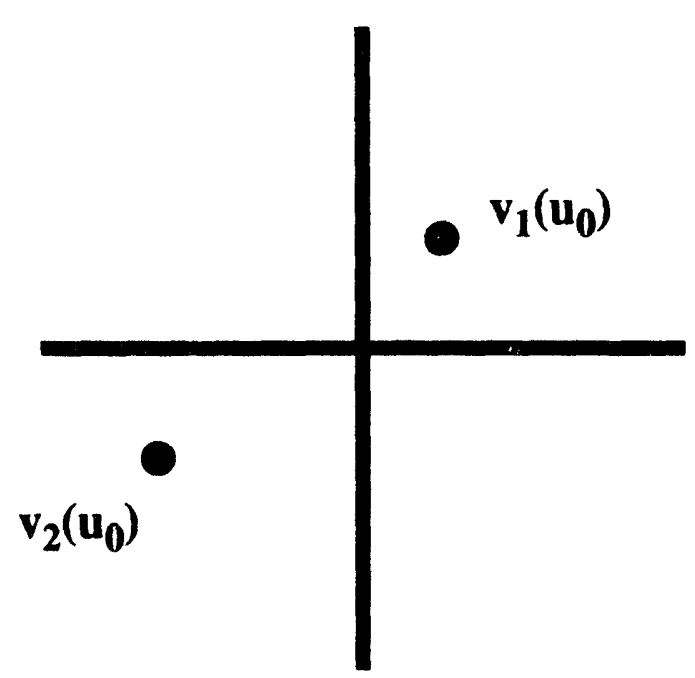

Figure 1. Typical rendition of $u$ space and $v$ space for a complex quadratic polynomial.

In their original work ${ }^{1}$ Lane and Bates would move $u$ in a series of increasing radius circles and plot the corresponding contours of the roots $v_{i}(u)$ as a means of visualizing the zero sheets. For low-degree polynomials (i.e., 2 or 3 ), it was easy to see that the zero sheet of the transform of a multiply-convolved image was the union of the zero sheets representing the individual trar 3 form factors. From the knowledge that the Fourier transform of an image of compact support is analytic, the zero sheet must be a smooth continuous function of the complex variable $u$. When individual zero sheets intersected one another, the intersection was not smooth (i.e., discontinuous derivative), and the separation was evident. For higher-degree polynomials (corresponding to larger, more realistic-sized images), zero-sheet visualization fails because of complexity. The purpose of zero-sheet visualization was to determine smoothness or analyticity at points of intersection so that separation could be invoked to group roots together that belonged to the same factor. Since this visualization fails for larger images, we decided to take a more direct approach to determine how the roots were grouped.

For simplicity let us choose an initial value of $u$ as the first Fourier point on the unit circle, $u=(1.0,0.0)$, and obtain the roots of the resulting polynomial in Eq. (6) 
by using a standard root-finding code. The location of the roots might resemble that shown in Figure 2 for a polynomial of degree 8.
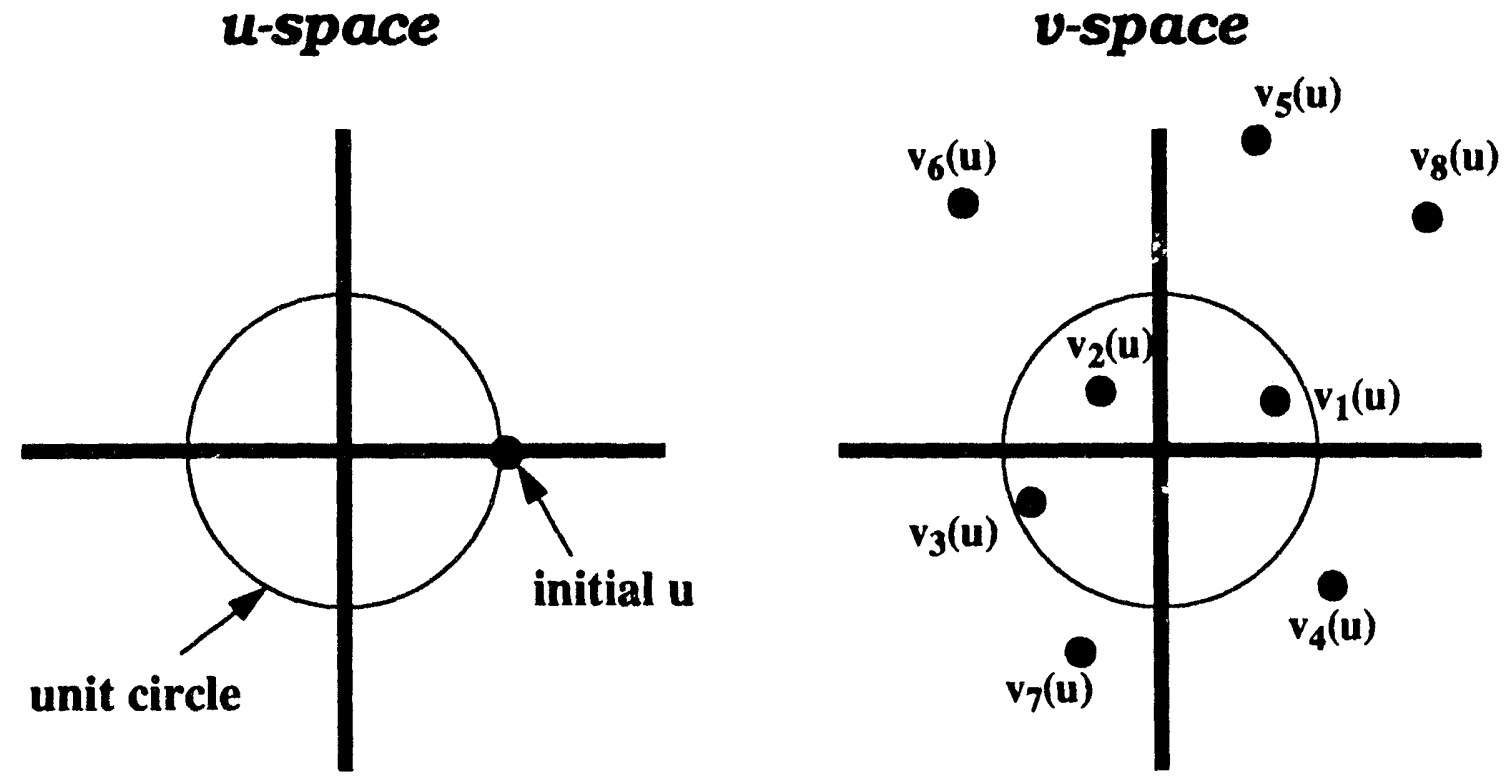

Figure 2. Typical root distribution resulting from specific $u$.

From this initial root distribution it is not yet possible to determine how many distinct factors (zero sheets) exist or which roots belong to which factor. Using our free parameter $u$, we are able to move $u$ in a mathematically determined path such that any pair of roots, $v_{i}$ and $v_{j}, i \neq j$, can be driven together. Therefore when $u$ reaches some $u_{0}, v_{j}\left(u_{0}\right)=v_{j}\left(u_{0}\right)$. A simple test for analyticity at this converged point $\left(u_{0}, v_{0}\right)$ will determine if $v_{i}(u)$ and $v_{j}(u)$ came together smoothly and therefore, perhaps, they belong on the same zero sheet or whether they came together via crossing paths and do not lie on the same zero sheet.

Given $N$ roots, there are $N(N-1) / 2$ pairs to be driven together, which offers a high degree of redundancy in determining which roots are associated with which. We will elaborate on this redundancy as a possible means of mitigating the effects of noise later in this paper.

A $u$ path can almost always (in the measure theoretic sense) be found that will drive any pair of roots together. We will now show how this path is determined. Let $u(s)$ be a path in the complex $u$ plane that is parameterized by the parameter $s$. Suppose that $v_{i}[u(s)]$ and $v_{j}[u(s)]$ are distinct roots to the equation $F(u, v)=0$, satisfying $F\left[u(s), v_{i}(s)\right]=0$, and $F\left[u(s) v_{j}(s)\right]=0$. Differentiating these equations with respect to $s$ yields

$$
\begin{aligned}
& F_{u}\left[u(s), v_{i}(s)\right] \dot{u}(s)+F_{v}\left[u(s), v_{i}(s)\right] \dot{v}_{i}(s)=0 \\
& F_{u}\left[u(s), v_{j}(s)\right] \dot{u}(s)+F_{v}\left[u(s), v_{j}(s)\right] \dot{v}_{j}(s)=0
\end{aligned}
$$


where

$$
F_{u}=\frac{\partial F}{\partial u}, F_{v}=\frac{\partial F}{\partial v}, \dot{u}=\frac{d u}{d s}, v=\frac{d v}{d s}
$$

and $s$ refers to arc length.

Solving for $\dot{v}_{i}$ and $\dot{v}_{j}$ above yields,

$$
\begin{aligned}
& v_{i}=\frac{-F_{u}\left(u, v_{i}\right) \dot{u}}{F_{v}\left(u, v_{i}\right)}, \\
& v_{j}=\frac{-F_{u}\left(u, v_{j}\right) \dot{u}}{F_{v}\left(u, v_{i}\right)} .
\end{aligned}
$$

Now we define the squared magnitude distance between roots $v_{i}$ and $v_{j}$ as

$$
D^{2}=\left|v_{i}-v_{j}\right|^{2}=\left(v_{i}-v_{j}\right)\left(v_{i}^{*}-v_{j}^{*}\right),
$$

where * denotes complex conjugate.

Since we want to choose a $u$ path that always causes roots $v_{i}$ and $v_{i}$ to come closer together, $D^{2}$ must be made a decreasing function of $u$. Therefore differentiating Eq. (18) with respect to $s$ yields

$$
\frac{d}{d s} D^{2}=2 \operatorname{Re}\left[\left(v_{i}^{*}-v_{j}^{*}\right)\left(\dot{v}_{i}-\dot{v}_{j}\right)\right]
$$

We wish to make Eq. (19) the most negative value possible (by selecting the proper direction for $u$ ) so that the selected roots come together as quickly as possible. From Eqs. (16) and (17) we see that

$$
\left(\dot{v}_{i}-\dot{v}_{j}\right)=\left[\frac{F_{u}\left(u, v_{j}\right)}{F_{v}\left(u, v_{i}\right)}-\frac{F_{u}\left(u, v_{i}\right)}{F_{v}\left(u, v_{i}\right)}\right] \dot{u} .
$$

Substitution of Eq. (20) into Eq. (19) yields

$$
\frac{d}{d s} D^{2}=2 \operatorname{Re}\left\{\left(v_{i}^{*}-v_{j}^{*}\right)\left[\frac{F_{u}\left(u, v_{j}\right)}{F_{v}\left(u, v_{j}\right)}-\frac{F_{u}\left(u, v_{i}\right)}{F_{v}\left(u, v_{i}\right)}\right] \dot{u}\right\}
$$

To make Eq. (21) the most negative value possible, we compute

$$
\left(v_{i}^{*}-v_{j}^{*}\right)\left[\frac{F_{u}\left(u, v_{j}\right)}{F_{v}\left(u, v_{j}\right)}-\frac{F_{u}\left(u, v_{i}\right)}{F_{v}\left(u, v_{i}\right)}\right]=\rho_{0} \exp \left(j \varphi_{0}\right) .
$$

If we substitute $\dot{u}=|\dot{u}| \exp (j \theta)$ into Eq. (21) and use the result from Eq. (22), we see that Eq. (21) is the most negative when $\phi_{0}+\theta=\pi$. Therefore the optimal angle is $\theta=\pi-\phi_{0}$, and the optimal direction in $u$ is $\dot{u}=|\dot{u}| \exp (j \theta)$. Iterations of this procedure define the optimal path to drive roots $v_{i}$ and $v_{j}$ together. 
We can always find a good step $\Delta u$ from

$$
\Delta u=|\dot{u}| \exp \left[j\left(\pi-\varphi_{0}\right)\right] \Delta s,
$$

where

$$
\phi_{0}=\text { Phase }\left\{\left(v_{i}^{*}-v_{j}^{*}\right)\left[\frac{F_{u}\left(u, v_{j}\right)}{F_{v}\left(u, v_{i}\right)}-\frac{F_{u}\left(u, v_{i}\right)}{F_{v}\left(u, v_{i}\right)}\right]\right\} \text {, }
$$

provided that $v_{i}^{*}-v_{j}^{*} \neq 0$ (which does not matter because this implies that the roots are already together) or that

$$
\left[\frac{F_{u}\left(u, v_{j}\right)}{F_{v}\left(u, v_{j}\right)}-\frac{F_{u}\left(u, v_{i}\right)}{F_{v}\left(u, v_{i}\right)}\right] \neq 0
$$

We emphasize that pathological examples exist in which two roots cannot be driven together. One such example is when $F(u, v)=\left(v-v_{1}\right)\left(v-v_{2}\right)$. Clearly, we cannot drive these roots together no matter how we deform the path in the $u$ plane because the roots are not a function of $u$ in this contrived case. We can say that, generically (in the measure theoretical sense), any two roots can be driven together. Since Eq. (25) is complex, it is extremely unlikely that both the real and imaginary parts would vanish simultaneously at any point along a 1-D curve in the $u$ plane. We admit that this argument is plausible but not rigorous. We appeal to the fact that we have never found a real example (other than contrived ones generated to illustrate the point) in which two roots could not be driven together. If we were to add almost any perturbation to our contrived examples, the roots could then be driven together.

It is worth noting that throughout the entire algorithm we will be computing many polynomial evaluations and first and second partial derivatives of these polynomials. Even though a simple code exists for simultaneous evaluation of a polynomial and a number of derivatives given the coefficients, ${ }^{9}$ several numerical tricks are employed to ensure stability of the evaluations. We must ensure that the polynomial does not blow up numerically when evaluating it for large-magnitude $u$ or $v$, for instance. We selectively reverse the order of the coefficients ${ }^{9}$ to be using reciprocal values of $u$ and/or $v$ effectively when those magnitudes are greater than unity; otherwise, we use normal ordering.

We therefore have four cases to consider for all polynomial and derivative evaluations:

$$
\begin{aligned}
& \text { Case 1: }|u| \leq 1.0,|v| \leq 1.0 \\
& \text { Case 2: }|u| \leq 1.0,|v|>1.0 \\
& \text { Case 3: }|u|>1.0,|v| \leq 1.0 \\
& \text { Case 4: }|u|>1.0,|v|>1.0
\end{aligned}
$$

It can be somewhat tedious to ensure mathematical robustness when computing polynomials and their derivatives for continuously changing values of $u$ and $v$.

Recall that Eq. (22) was used to find the optimal direction of the step, not the magnitude. The step, $\Delta u$, is defined as $\Delta u=\dot{u} \Delta s$. We make an initial estimate of what the maximum magnitude step size $|\Delta u|_{\text {max }}$ should be by expanding the change in $v$ by a second-order Taylor series: 


$$
v+\Delta v \cong v+\frac{d v}{d u} \Delta u+\frac{1}{2} \frac{d^{2} v}{d u^{2}}(\Delta u)^{2} .
$$

Keeping the ratio of the magnitudes of the quadratic and linear terms to less than 0.1 (i.e., a small value), we obtain a first estimate of $|\Delta u|_{\text {max }}$ :

$$
\frac{\frac{1}{2}\left|v_{u u}\right|\left(|\Delta u|_{\max }\right)^{2}}{\left|v_{u}\right||\Delta u|_{\max }} \leq 0.1
$$

Rearranging relationship (27) yields

$$
|\Delta u|_{\max } \leq \frac{0.1\left|v_{u u}\right|}{0.5\left|v_{u}\right|}
$$

This simple test was found to be inadequate when the root we were tracking came extremely close to another root that was on a different zero sheet. In this case, relationship (28) did not ensure that the step size was small enough that we could avoid getting the two roots mixed up. We developed the following additional test, which managed to avoid these mixups. Expanding $F$ in a second-order Taylor series, we obtain

$$
F_{u} \Delta u+F_{v} \Delta v+\frac{1}{2}\left[F_{u u}(\Delta u)^{2}+2 F_{u v} \Delta u \Delta v+F_{v v}(\Delta v)^{2}\right]=0
$$

For any value of $\Delta u$ we obtain two solutions for $\Delta v$. When two roots on different zero sheets are extremely close to each other, both $F_{\mu}$ and $F_{\nu}$ are small. If the roots actually came together, $F_{u}$ and $F_{v}$ would vanish, and the two solutions for $\Delta v$ from Eq. (29) would give good approximations to the two possible solutions at a nearby value of $u$. When we are near an intersection point (root path crossing point), the solutions of Eq. (29) will still give a good approximation to the two possible roots only if $\Delta u$ is not too small. However, as $\Delta u \rightarrow 0$, one of the solutions to Eq. (29) approaches infinity and the other solution remains as a good approximation to the root position that we are predicting. Therefore, if we choose $\Delta u$ values that are small enough, the ratio of the magnitudes of the two solutions can be made arbitrarily large. We implement this procedure as follows.

For our given $\Delta u$ [found from relations (23) and (28)], we solve Eq. (29) for the two solutions of $\Delta v / \Delta u$ by solving the quadratic

$$
\left(F_{u}+\frac{1}{2} F_{u u} \Delta u\right)+\left(F_{v}+2 F_{u v} \Delta u\right)\left(\frac{\Delta v}{\Delta u}\right)+F_{\nu v}\left(\frac{\Delta v}{\Delta u}\right)^{2}=0
$$

If the two solutions $(\Delta v / \Delta u)_{1}$ and $(\Delta v / \Delta u)_{2}$ are markedly different from each other (i.e., the ratio of their magnitudes is greater than 20), then the step size $|\Delta u|_{\max }$ is small enough and the process of root driving can proceed for one step. If the two solutions do not differ much, $|\Delta u|_{\text {max }}$ is repeatedly subdivided by 2 until the solutions of Eq. (30) differ substantially. The new root locations are predicted with 
the use of Eq. (26) and then polished to the precise locations using Laguerre's rootpolishing technique. ${ }^{8}$

This method of step size determination has always provided a robust solution, never taking too large a step, which would permit root positions to change too radically and thus increase the likelihood of root mixups, or too small a step, which would waste computer time. This method is also employed in the root-tracking process discussed in subsection 4.2.

Once roots $v_{i}$ and $v_{j}$ have been driven to within some predetermined distance of each other, $\left|v_{i}-v_{i}\right| \leq 0.01$, it is much more efficient and robust to use Newton's method to drive the roots completely together. When two roots come together at $\left(u_{0}, v_{0}\right)$ and form a double root of $F\left(u_{0}, v_{0}\right)$, it is also true that $F_{v}\left(u_{0}, v_{0}\right)=0$. Thus we have the two simultaneous equations that are solved by Newton's method:

$$
\begin{aligned}
& F(u, v)=0 \\
& F_{v}(u, v)=0
\end{aligned}
$$

Relations (31) and (32) are solved twice, once using the value of $u_{i}$ and $v_{i}$ as the initial guess, then by using the value of $u_{j}$ and $v_{j}$ as the initial gress. If Newton's method converges, both convergence points (solutions) $\left(u_{0}, v_{0}\right)$, and $\left(u_{0}, v_{0}\right)$ will be the same to within machine precision. A typical $u$ path and $v$ paths for a pair of roots are shown in Figure 3.

\section{u-space}

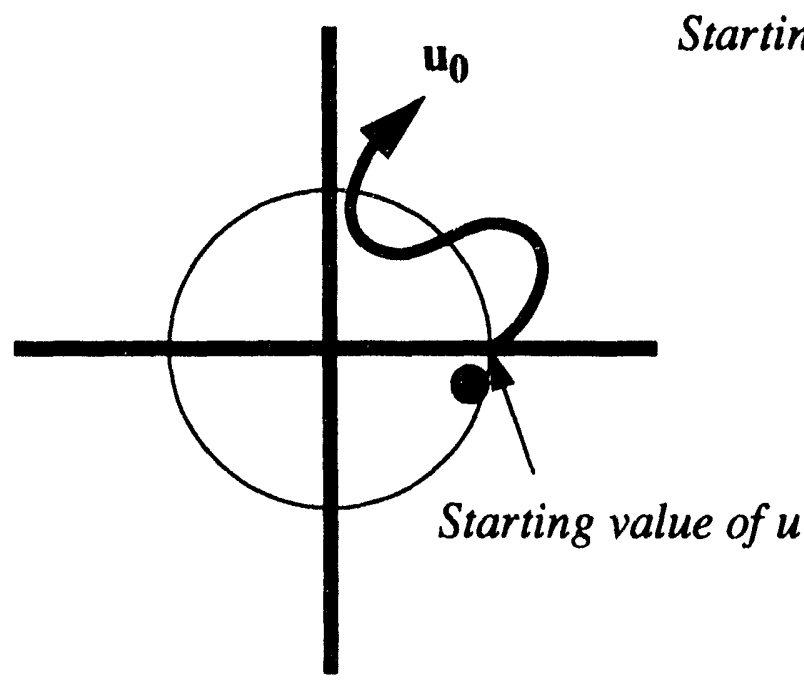

v-space

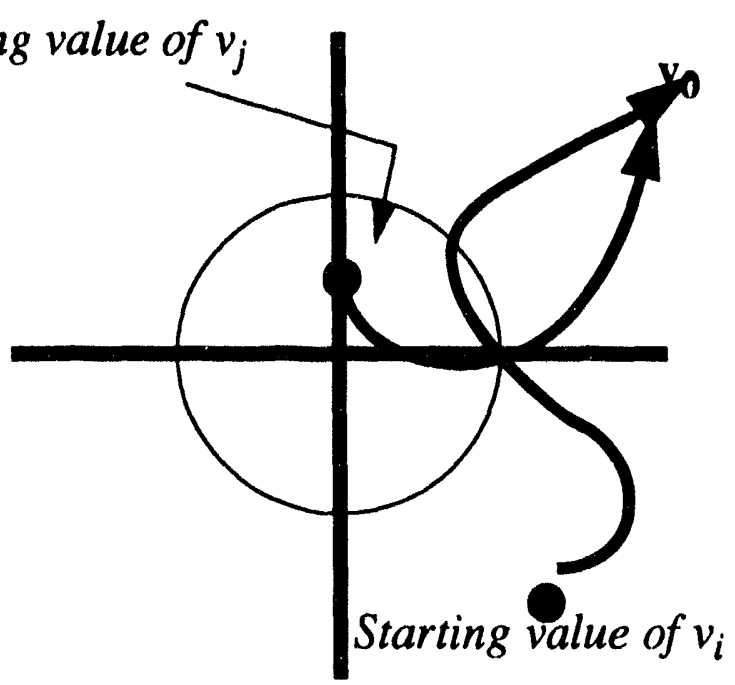

Figure 3. Typical $u$ path causing roots $v_{i}$ and $v_{j}$ to coverge at some point $v_{0}$.

Now that we have the precise point $\left(u_{0}, v_{0}\right)$ where roots $v_{i}$ and $v_{j}$ converged, we test for analyticity and the strength of association between roots $v_{i}$ and $v_{j}$. We would like the coupling strength to be near unity if roots $v_{i}$ and $v_{j}$ are on the same zero sheet and near zero if they are on different zero sheets. Theoretically $F_{\mu}=0$ at the convergence point. In practice it is not possible to make such a precise statement because noise would destroy this condition. It is also not a good idea to merely require that $\left|F_{u}\right|<\delta$, where $\delta$ is some small number, because this test would not be 
scale invariant. Using the fact that the system of equations given by Eqs. (31) and (32) is singular when $F_{\mu}=0$ suggests that a scale-invariant measure can be found by using an eigenvalue approach.

We have settled on the following measure to determine coupling strengths between roots. At $\left(u_{0}, v_{0}\right)$ we compute the two eigenvalues of the following Jacobian matrix and select the largest magnitude eigenvalue $|\lambda|_{\max }$ :

$$
J=\left[\begin{array}{ll}
F_{u u} & F_{u v} \\
F_{u v} & F_{v v}
\end{array}\right] .
$$

Our coupling strength between roots $v_{i}$ and $v_{j}$ is defined as

$$
S(i, j)=\frac{\left|F_{u}\left(u_{0}, v_{0}\right)\right|}{|\lambda|_{\max }} .
$$

The $S(i, j)$ form an $N \times N$ symmetric real matrix ( $N$ is the number of roots) whose entries reflect the strength of association between roots indexed $i$ and $j$.

If the two sets of convergence points from the solution of relations (31) and (32) are not within machine precision, it is most likely due to Newton's method not converging. Fortunately this seems to happen only if roots $v_{i}$ and $v_{j}$ do not lie on the same zero sheet, and thus we obtain by default a coupling strength of zero, precisely what we want for this condition. Remember, for numerical stability we always account for the four cases of $u$ and $v$ magnitudes mentioned above, in root driving, in Newton's method, and in coupling strength determination.

All the information about root associations is contained in the association matrix $S$. For noiseless data, entries in $S$ will be near unity for roots that are associated; for roots that are not associated, such entries will be near or equal to zero. It is a simple matter to threshold the matrix to determine precisely the number of different root groups that exist and which roots belong in each group. A typical example will illustrate this clearly.

Let us suppose that we drive the $N(N-1) / 2$ pairs of roots together and compute their coupling strength, the $S$ matrix is given below for the case of eight roots. Since a root is always perfectly associated with itself, $S$ contains 1.0 along the diagonal:

$$
S=\left[\begin{array}{cccccccc}
1 & 0.12 & 0 & 0 & 0 & 0.34 & 0 & 0 \\
0.12 & 1 & 0 & 0 & 0 & 0.25 & 0 & 0 \\
0 & 0 & 1 & 0.22 & 0 & 0 & 0.18 & 0 \\
0 & 0 & 0.22 & 1 & 0 & 0 & 0.16 & 0 \\
0 & 0 & 0 & 0 & 1 & 0 & 0 & 0.42 \\
0.34 & 0.25 & 0 & 0 & 0 & 1 & 0 & 0 \\
0 & 0 & 0.18 & 0.16 & 0 & 0 & 1 & 0 \\
0 & 0 & 0 & 0 & 0.42 & 0 & 0 & 1
\end{array}\right]
$$

Thresholding $S$ at a value of say $10^{-5}$, we obtain the thresholded $S$ matrix 


$$
\bar{S}=\left[\begin{array}{llllllll}
1 & 1 & 0 & 0 & 0 & 1 & 0 & 0 \\
1 & 1 & 0 & 0 & 0 & 1 & 0 & 0 \\
0 & 0 & 1 & 1 & 0 & 0 & 1 & 0 \\
0 & 0 & 1 & 1 & 0 & 0 & 1 & 0 \\
0 & 0 & 0 & 0 & 1 & 0 & 0 & 1 \\
1 & 1 & 0 & 0 & 0 & 1 & 0 & 0 \\
0 & 0 & 1 & 1 & 0 & 0 & 1 & 0 \\
0 & 0 & 0 & 0 & 1 & 0 & 0 & 1
\end{array}\right]
$$

The number of distinct row or column vectors in $\bar{S}$ determines the number of roots groups [factors of $F(u, v)$ ] and the indices of the nonzero entries are the indices of roots that belong in the same group. It is easy to see from $\bar{S}$ that there are three distinct row vectors:

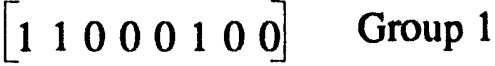

$$
\begin{aligned}
& {\left[\begin{array}{llllllll}
0 & 0 & 1 & 1 & 0 & 0 & 1 & 0
\end{array}\right] \quad \text { Group } 2} \\
& {\left[\begin{array}{llllllll}
0 & 0 & 0 & 0 & 1 & 0 & 0 & 1
\end{array}\right] \quad \text { Group } 3}
\end{aligned}
$$

Thus roots 1, 2, and 6 belong to group 1, roots 3,4 , and 7 belong to group 2 , and roots 5 and 8 belong to group 3 . At this point we know that $F(u, v)$ is factorizable into three distinct factors, and we know which roots belong to those factors for our initially chosen $u$ at $(1,0)$. Also note that the three row vectors are mutually orthogonal.

Diagrammatically, $u$ space and $v$ space with the now known root groupings is shown in Figure 4.

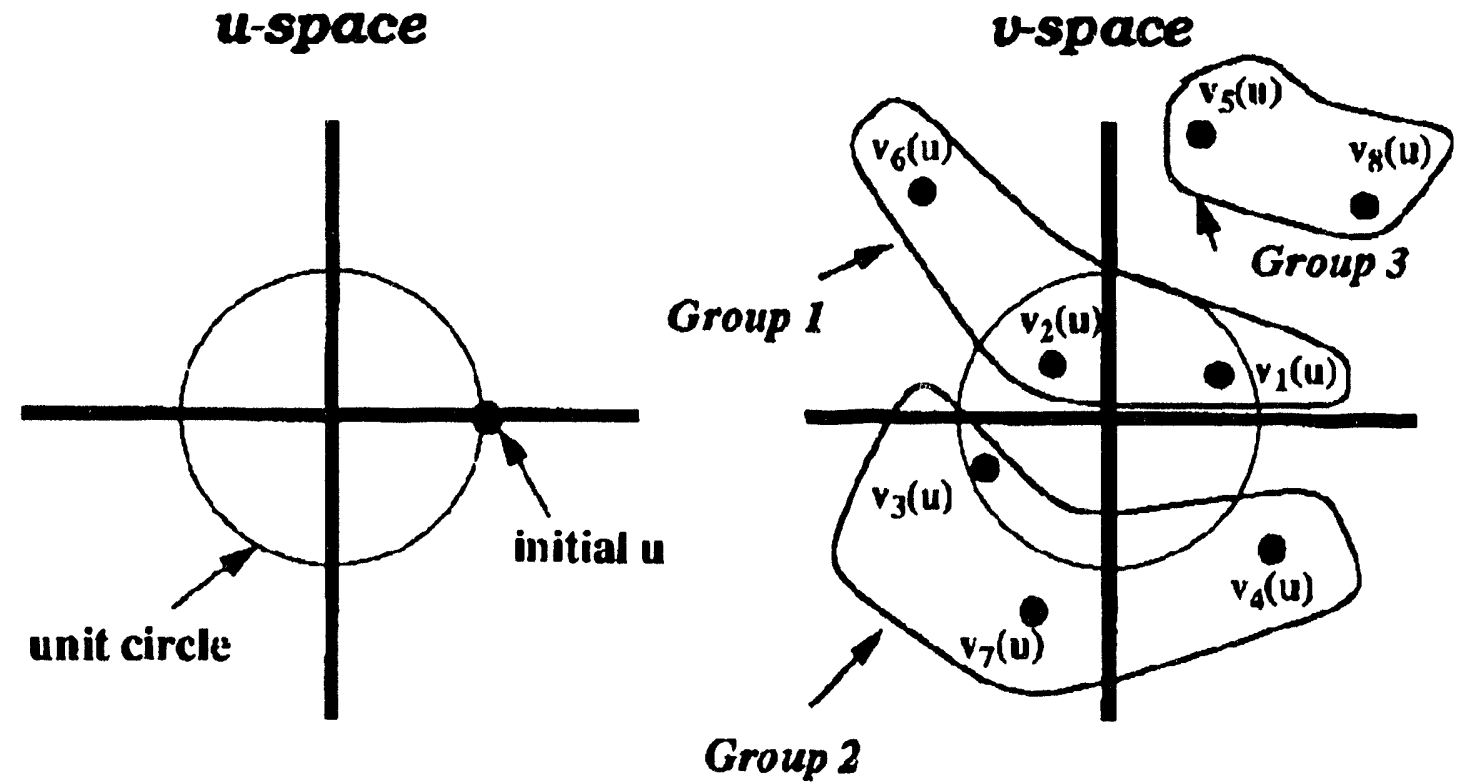

Figure 4. Typical root grouping. 
The addition of noise to $F(u, v)$ almost always destroys the factorizability in theory. In practice a small amount of noise changes the entries in $S$. More specifically, all the zero-valued entries become small nonzero values whose magnitude depends on the amount of added noise. If the amount of noise is small, $S$ can probably still be thresholded to invoke the true partitioning. If $S$ cannot be thresholded, the redundancy of associations and their strengths can almost certainly be exploited to invoke some best-sense partitioning. We are currently investigating some simple operations that exploit the dot product (orthogonality) properties of the thresholded matrix vectors to correct a few random association errors caused by noise or numerical rounding errors.

An error can be made, for example, if while driving roots $v_{i}$ and $v_{j}$ are driven together another root $v_{k}$ comes so close to either $v_{i}$ or $v_{j}$ that our prediction of the new position of $v_{i}$ or $v_{j}$ jumps onto $v_{k}$ and we go on merrily with the wrong pair of roots and ultimately the wrong association.

During root driving, we do not now deviate the $u$ path deviate to avoid other roots coming close to either root in the pair of interest, although we could do so. We do, however, use path-deviation techniques during root tracking, as we will discuss in subsection 4.2.

We are also investigating more sophisticated graph-theoretical methods that exploit the weights and redundancies in the full $S$ matrix to invoke a best partitioning. For the noiseless (except for numerical rounding errors) case of interest in this paper, we can almost always correct a few errors automatically and obtain the correct factorization.

Computationally, the root-driving part of the algorithm is the most time consuming. There are $O\left(N^{2}\right)$ pairs of roots to be driven together (for full redundancy, which is an advantage when dealing with noise), and there are $O\left(N^{2}\right)$ operations per pair of roots. Therefore the root-driving process is $O\left(N^{4}\right)$.

\subsection{Root Tracking and Fourier-Transform Factor Generation}

Once the roots are correctly grouped, it is necessary to track each root correctly while $u$ moves in small steps sequentially to each Fourier point on the unit circle, $u_{k}=\exp (j 2 \pi k / M), k=0,1,2, \ldots M-1$. This tracking operation maintains the integrity of each transform factor and permits us to build up each transform factor one column at a time. The columns of factor $A_{i}(u, v)$ are constructed by using all the roots for factor $A_{i}(u, v)$ according to the following equation

$$
A_{i}(u, v)=\prod_{m=1}^{N R}\left[v_{n}-v_{m}\left(u_{k}\right)\right]
$$

where $u_{k}=\exp (-j 2 \pi k / M), k=0,1,2, \ldots, M-1 ;\left(\nu_{n}=\exp (-j 2 \pi n / N), n=0\right.$, $1,2, \ldots, N-1)$; NR is the number of roots for group i, and $v_{m}\left(u_{k}\right)$ is root $m$ of group $i$; for $u=u_{k}$.

The $A$ in $A_{i}(u, v)$ refers to the factor being built up columnwise. When the transform factors are built up rowwise after root driving and association with the transposed input data, we will use the designator $B_{j}(u, v)$. After all factors are constructed both columnwise and rowwise, the $A_{i}$ and $B_{j}$ matrices must be properly paired up and the arbitrary row/column complex scalings removed. This matrix- 
equalization step is the final part of the algorithm and will be discussed in subsection 4.3.

Although root tracking is much less time consuming than root driving $\left[O\left(N^{2}\right)\right.$ versus $O\left(N^{4}\right)$, respectively], the root-tracking part of the algorithm was the most difficult and most error prone (from a numerical precision point of view); thus it required the most care in numerical implementation. Let us begin the discussion with all the roots properly grouped for the value of $u$ being the first point of interest on the unit circle, $u_{0}=(1,0)$. We wish to move $u$ in small steps toward $u_{1}=\exp (j 2 \pi / M)$ and ensure that all our carefully grouped roots do not get mixed up. Root mixups are possible when two roots (say, $v_{k}$ and $v_{l}$, each in a different group) come quite close to each other. If $v_{k}$ and $v_{l}$ are close, it is possible that their predicted positions swap and converge to the opposite roots. Thus, as we track $v_{k}$ in group $i$, it suddenly becomes $v_{l}$ in group $j$ without our knowledge. The integrity of the root groupings is lost, and the proper factorization has been altered. We were never able to avoid root mixups by just reducing the step size; therefore, a more sophisticated approach was adopted.

To avoid the possibility of root mixups, it is necessary to choose a $u$ path that accomplishes two major objectives. First, we must follow a path that keeps any pair of roots from getting too close, and second, we must be guaranteed that $u$ eventually (and efficiently) gets to its desired location.

The implicit function theorem of calculus indicates that $F_{v}=0$ for any $v_{l}=v_{i}$. When moving $u$ around the unit circle, we wish to avoid those values of $u$ for which $F_{y}$ becomes too small. We define a path that permits us to choose an optimal direction for $u$ while simultaneously ensuring that $F_{v}$ does not become too small and that $u$ will eventually reach its desired location, $u=u_{\uparrow}$. To accomplish this we always wish to take a step of magnitude $|\Delta u|$ in a direction where $\left|F_{v}\right|^{2}$ increases, subject to the constraint that the given step $\Delta u$ takes us closer to $u_{d}$. The diagram shown in Figure 5 should help delineate the strategy.

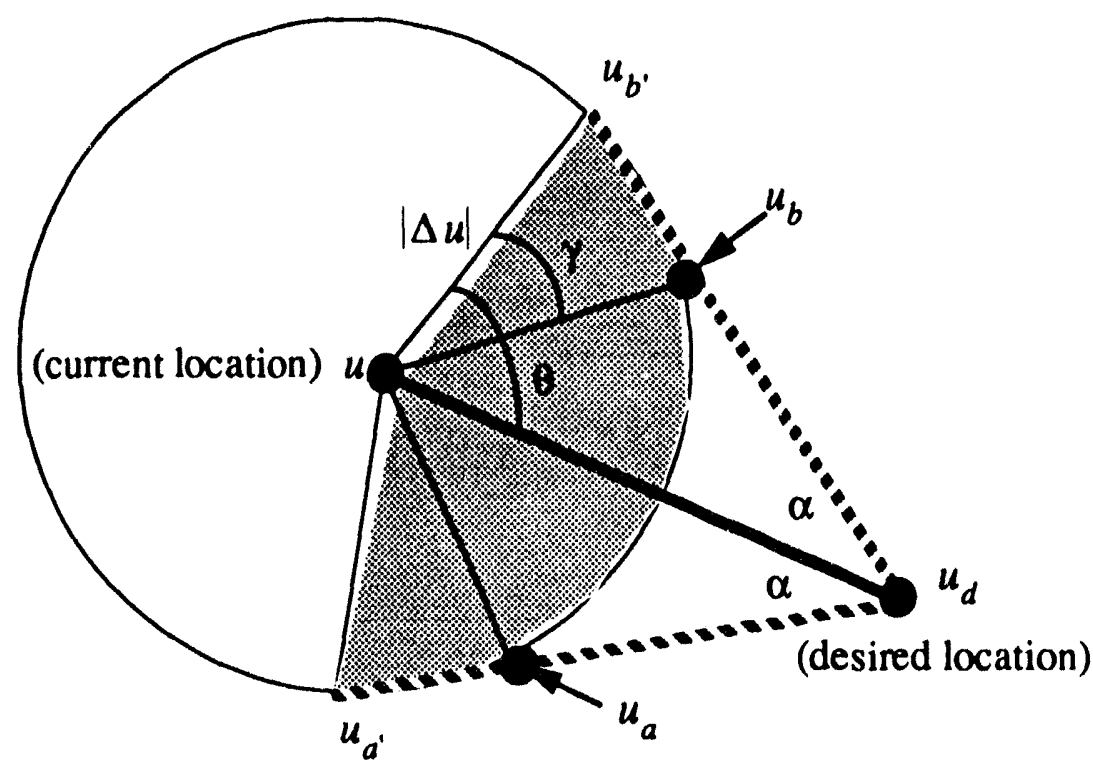

Figure 5. Geometrical rendition of variable $u$ path step. 
The optimal direction for $|\Delta u|$ is found by making $\Delta\left|F_{v}\right|^{2}$ the most positive value possible. Mathematically,

$$
\Delta\left|F_{v}\right|^{2}=2 \operatorname{Re}\left[F_{\nu}^{*}\left(F_{u \nu}+F_{\nu \nu} v_{u}\right) \Delta u\right]
$$

Letting $F_{\psi}^{*}\left(F_{u v}+F_{v v} v_{u}\right)=A \exp (j \psi)$ and $\Delta u=B \exp (j \phi)$ in Eq. (41), we now see that $\Delta\left|F_{v}\right|^{2}$ is the most positive when $\psi+\phi=0$; therefore, $\phi=-\psi$ is the optimal direction. The step size $|\Delta u|$ is determined by the same methods described in subsection 4.1 above.

If $u+|\Delta u| \exp [j \phi]$ puts us inside the shaded region shown in Figure 5, then we take that step. Otherwise we step to either $u_{a}$ or $u_{b}$, depending on where the predicted value of $\left|F_{y}\right|^{2}$ is larger. This strategy forces us to make some progress toward $u_{d}$. The angle $\gamma$, which is somewhat arbitrary, rotates the step well inside the shaded region and is currently set at $3 \pi / 16 \mathrm{rad}$ (not so small that virtually no progress is made toward $u_{d}$ but also not so large that we step so directly towards $u_{d}$ that the roots actually come closer together than before).

Each root is tracked individually with $u$ moving from the current point on the unit circle to the next desired value of $u$. Once all the tracked roots are available at the new $u$, they can be compared with the roots obtained (using a standard root solver) from the original polynomial at that particular value of $u$. This procedure permits us to obtain a check on the incegrity of the tracked roots versus those obtained with a root solver. We could not, of course, just obtain the roots at each desired $u$ with a root solver because we would be unable to tell which roots belonged to which group. The tracking procedure maintains the root groupings.

There is a subtle but potentially disastrous problem that occurs during root tracking. Since each root is tracked individually, the adaptive $u$ path is tuned to the particular root we are tracking. This means that the roots are tracked with different $u$ paths. The extremely complicated topology of the zero sheets allows for the possibility of roots lying on the same zero sheet to be tracked to a common point if different $u$ paths are taken. For example, if $v_{1}(u), v_{2}(u)$, and $v_{3}(u)$ all belong to the same group and they are tracked from $u=u_{0}$ to $u=u_{1}$ with different paths, it is possible that, say, $v_{1}\left(u_{0}\right)$ goes to $v_{1}\left(u_{1}\right), v_{2}\left(u_{0}\right)$ goes to $v_{1}\left(u_{1}\right)$, and $v_{3}\left(u_{0}\right)$ goes to $v_{3}\left(u_{1}\right)$. Therefore roots $v_{1}$ and $v_{2}$ converged to the same redundant root, and the integrity of the group was lost. We can of course check for this redundancy by a one-to-one comparison with the true roots from a root solver and determine which roots are in error. The fix is to go back and retrack roots $v_{1}$ and $v_{2}$ simultaneously by using a common $u$ path for both roots.

Simultaneous root tracking is accomplished by defining a $u$ path that depends on sums of the partial derivatives for the roots concerned instead of just individual partials. These sums of partials are used in an equation such as Eq. (41), and a common $u$ path is developed. Each time a root or multiple roots are tracked to the desired $u$, all roots are again checked for redundancy by comparison to the root-solver values, and any new or additional redundant roots are retracked. This procedure is repeated until all the tracked roots agree in a one-to-one correspondence to the true roots from a root solver. It is then safe to begin tracking each root individually to the next desired value of $u$, repeating the above procedure until we have successfully tracked all the roots completely around the unit circle and loaded the columns of the individual transform matrices according to Eq. (40). 
In practice it is typical that only a small percentage of roots need to be retracked. Therefore it is computationally more efficient and numerically more stable to track individual roots and then recrack roots simultaneously as necessary than to track all roots simultaneously from the start.

\subsection{Transform Matrix Equalization and Deconvolved Image Formation}

Recall that both the root-driving and association step and the root-tracking step must be done twice, once with the original data, ultimately to load transform matrices $A_{i}$ columnwise, and once again with the transposed caata set to load transform matrices $B_{i}$ rowwise. The computation of a polynomial from its roots leaves an arbitrary complex scaling. Since each column and row of the transform matrices were constructed from evaluating a 1-D polynomial, each row and column has a different complex scaling. Also, since a particular $A_{i}$ represents the same transform factor as some $B_{i}$, except for the row/column scalings, the $A$ 's and $B$ 's must be properly paired up and the complex scalings removed before they can be inverse transformed to form the image-convolution components.

Let us assume that the matrix $A$ has a different complex factor multiplying the true underlying column elements, whereas the matrix $B$ has different row scalings. Thus

$$
\begin{aligned}
& A_{i j}=c_{j} a_{i j}, \\
& B_{i j}=d_{i} a_{i j},
\end{aligned}
$$

and the problem is to determine the $a_{i j}$ 's from $A$ and $B$. From Eqs. (42) and (43) we see that

$$
d_{i} A_{i j}=c_{j} B_{i j}
$$

and the set of equations that must be solved is

$$
d_{i} A_{i j}-c_{j} B_{i j}=0 .
$$

Eq. (45) results in a matrix equation

$$
\Gamma x=0,
$$

where

$$
x=\left[\begin{array}{llllllll}
d_{1} & d_{2} & \ldots & d_{M} & c_{1} & c_{2} & \ldots & c_{N}
\end{array}\right]^{T}
$$

and 


$$
\Gamma=\left[\begin{array}{cccccccccc}
A_{11} & 0 & 0 & \ldots & 0 & -B_{11} & 0 & 0 \ldots & 0 \\
0 & A_{21} & 0 & \ldots & 0 & -B_{21} & 0 & 0 \ldots & 0 \\
0 & 0 & A_{32} & \ldots & 0 & -B_{31} & 0 & 0 \ldots & 0 \\
0 & 0 & 0 & \ldots & 0 & \ldots & 0 & 0 \ldots & 0 \\
0 & 0 & 0 & \ldots & A_{M 1} & -B_{M 1} & 0 & 0 & \ldots & 0 \\
A_{12} & 0 & 0 & \ldots & 0 & 0 & -B_{12} & 0 \ldots & 0 \\
0 & A_{22} & 0 & \ldots & 0 & 0 & -B_{22} & 0 \ldots & 0 \\
0 & 0 & A_{33} & \ldots & 0 & 0 & -B_{32} & 0 \ldots & 0 \\
0 & 0 & 0 & \ldots & 0 & 0 & \ldots & 0 & \ldots & 0 \\
0 & 0 & 0 & \ldots & A_{M 3} & 0 & -B_{M 2} & 0 & \ldots & 0 \\
A_{1 N} & 0 & 0 & \ldots & 0 & 0 & 0 & 0 & \ldots & -B_{1 N} \\
0 & A_{2 N} & 0 & \ldots & 0 & 0 & 0 & 0 & \ldots & -B_{2 N} \\
0 & 0 & A_{3 N} & \ldots & 0 & 0 & 0 & 0 \ldots & -B_{3 N} \\
0 & 0 & 0 & \ldots & 0 & 0 & 0 & 0 \ldots & \ldots \\
0 & 0 & 0 & \ldots & A_{M N} & 0 & 0 & 0 \ldots & -B_{M N}
\end{array}\right]
$$

In practice $A$ and $B$ will be inconsistent because of noise. In addition, since Eq. (46) is overdetermined, we must solve Eq. (46) in a least-squares sense by solving

$$
\Gamma^{T} \Gamma x=\lambda x, \quad x^{T} x=1
$$

for the eigenvector $x$ corresponding to the smallest eigenvalue $\lambda$ of $\Gamma^{T} \Gamma$.

We do not actually compute the matrix $\Gamma$ because of its size, but we do compute in closed form the matrix $\Gamma^{T} \Gamma$, which is only $(M+N) \times(M+N)$. We then find the eigenvector $x$ corresponding to the smallest eigenvalue and use the elements of $x$ to equalize the two matrices $A$ and $B$ and form a single transform matrix from the average of the two equalized matrices. Mathematically,

$$
C_{i j}=\frac{1}{2}\left[d_{i} A_{i j}+c_{j} B_{i j}\right]
$$

We try all combinations of the $A_{i}$ matrices [i $=1,2, \ldots$ (number of groups)] with the $B_{j}$ matrices $\left[\mathrm{j}=1,2, \ldots\right.$ (number of groups)] and choose the particular $A_{i}$ and $B_{j}$ that produce the smallest eigenvalue during the solution of Eq. (49). This allows us to properly pair up the appropriate column- and row-generated matrices that represent the same transform factor and remove the row/column scalings simultaneously. After 
equalization, there is only one overall arbitrary complex scaling multiplying the entire matrix, and it is of no consequence. The equalized transform matrices are then inverse transformed to yield the individual convolution components. The matrixequalization step is numerically robust and computationally only a small part of the blind deconvolution process.

A simple example will illustrate the equalization process. Let the unequalized matrices $A$ and $B$ be as follows.

$$
\begin{aligned}
& A=\left[\begin{array}{cc}
1 & 1 / 2 \\
3 & 1 \\
5 & 3 / 2 \\
7 & 2
\end{array}\right], \\
& B=\left[\begin{array}{cc}
1 & 2 \\
9 / 2 & 6 \\
10 & 12 \\
35 / 2 & 20
\end{array}\right]
\end{aligned}
$$

$A$ has different column scalings, and $B$ has different row scalings. The matrix $\Gamma$, which we normally do not compute but is illustrated for clarity, becomes

$$
\Gamma=\left[\begin{array}{cccccc}
1 & 0 & 0 & 0 & -1 & 0 \\
0 & 3 & 0 & 0 & -9 / 2 & 0 \\
0 & 0 & 5 & 0 & -10 & 0 \\
0 & 0 & 0 & 7 & -35 / 2 & 0 \\
1 / 2 & 0 & 0 & 0 & 0 & -2 \\
0 & 1 & 0 & 0 & 0 & -6 \\
0 & 0 & 3 / 2 & 0 & 0 & -12 \\
0 & 0 & 0 & 2 & 0 & -20
\end{array}\right] .
$$

Since in this example, $A$ and $B$ represent perfect but unequalized matrices, the smallest eigenvalue of $\Gamma^{T} \Gamma$ equals zero. The eigenvector $x$ associated with the zero eigenvalue is

$$
x=\left[\begin{array}{l}
0.2620 \\
0.3931 \\
0.5241 \\
0.6551 \\
0.2620 \\
0.0655
\end{array}\right]
$$


If we normalize $x$ by its first element, we find that

$$
x=\left[\begin{array}{c}
1.0 \\
1.5 \\
2.0 \\
2.5 \\
1.0 \\
0.25
\end{array}\right]=\left[\begin{array}{l}
d_{1} \\
d_{2} \\
d_{3} \\
d_{4} \\
c_{1} \\
c_{2}
\end{array}\right] .
$$

The equalized matrices, $A_{e}$ and $B_{e}$, are found from

$$
\begin{aligned}
& A_{e}=A=\left[\begin{array}{cc}
1 & 1 / 2 \\
3 & 1 \\
5 & 3 / 2 \\
7 & 2
\end{array}\right]\left[\begin{array}{cc}
1 / c_{1} & 0 \\
0 & 1 / c_{2}
\end{array}\right]=\left[\begin{array}{ll}
1 & 2 \\
3 & 4 \\
5 & 6 \\
7 & 8
\end{array}\right], \\
& B_{e}=\left[\begin{array}{cccc}
1 / d_{1} & 0 & 0 & 0 \\
0 & 1 / d_{2} & 0 & 0 \\
0 & 0 & 1 / d_{3} & 0 \\
0 & 0 & 0 & 1 / d_{4}
\end{array}\right]\left[\begin{array}{cc}
1 & 2 \\
9 / 2 & 6 \\
10 & 12 \\
35 / 2 & 20
\end{array}\right]=\left[\begin{array}{ll}
1 & 2 \\
3 & 4 \\
5 & 6 \\
7 & 8
\end{array}\right] .
\end{aligned}
$$

If $A$ and $B$ differed slightly because of noise or numerical differences, we would obtain a combined equalized matrix by averaging the two solutions.

\section{Algorithm Summary}

We believe that it is worthwhile to summarize briefly in words our systematic blind deconvolution procedure. The three major steps are

\subsection{Polynomial Root Association and Grouping}

(a) The 2-D polynomial corresponding to the Fourier transform of the multiplyconvolved image is represented as a $1-\mathrm{D}$ polynomial in the variable $v$ whose coefficients depend on the variable $u$.

(b) The roots of this 1-D polynomial are computed with a standard root solver using a particular value of $u$.

(c) The complex variable $u$ is moved along a mathematically derived path that sequentially causes all pairs of roots to merge at common points. 
(d) A simple test of analyticity at the point where a pair of roots converges determines the coupling strength of those roots and the likelihood of their belonging to the same transform factor.

(e) Tests for all pairs of roots generate a coupling strength or association matrix that defines which roots belong to which factors.

(f) At this point we know, for the first time, exactly how many images were convolved together to form the original.

\subsection{Root Tracking and Fourier-Transform Factor Generation}

(a) Once the roots have been grouped, we track the roots as the complex variable $u$ sequentially moves to each appropriate Fourier point on the unit circle.

(b) At each point on the unit circle, the columns of the Fourier-transform factor matrices are generated from the knowledge of the roots for each factor.

(c) On completion of traversing the unit circle, each 2-D transform factor has been filled columnwise.

(d) The original data are then transposed, and steps 5.1 and 5.2 above are again performed in their entirety to result in Fourier-transform factor matrices being built up rowwise.

(e) We thus have two sets of transform factor matrices, those built from columns and those built from rows.

\subsection{Transform Matrix Equalization and Deconvolved Image Formation}

(a) The two sets of transform matrices are associated and merged into a common set with the arbitrary complex row/column scalings removed.

(b) We have developed a robust eigenvalue/eigenvector method to perform this step.

(c) Once the transform matrices are equalized, the individual image-convolution components are formed by inverse Fourier transformation of each equalized transform matrix.

\section{Examples of Deconvolved Complex Imagery}

Figure 6(a) depicts the magnitude of a small multiply-convolved complex image magnified by pixel replication for display. By using only the data represented by Figure $6(a)$ as the input to the algorithm, we use the code to determine that there are two transform factors and to obtain the convolution components. The magnitudes of the deconvolved complex images are shown in Figures 6(b) and 6(c). These $8 \times 8$ pixel images, which consist of recognizable characters superimposed on a lower-level random background, are identical to the complex images that were originally convolved to yield the input depicted by Figure 6(a). For most tests the image components have recognizable magnitudes and random phases. Problems of this size are solved in $\sim 15$ min on a Sparc 2 work station. 


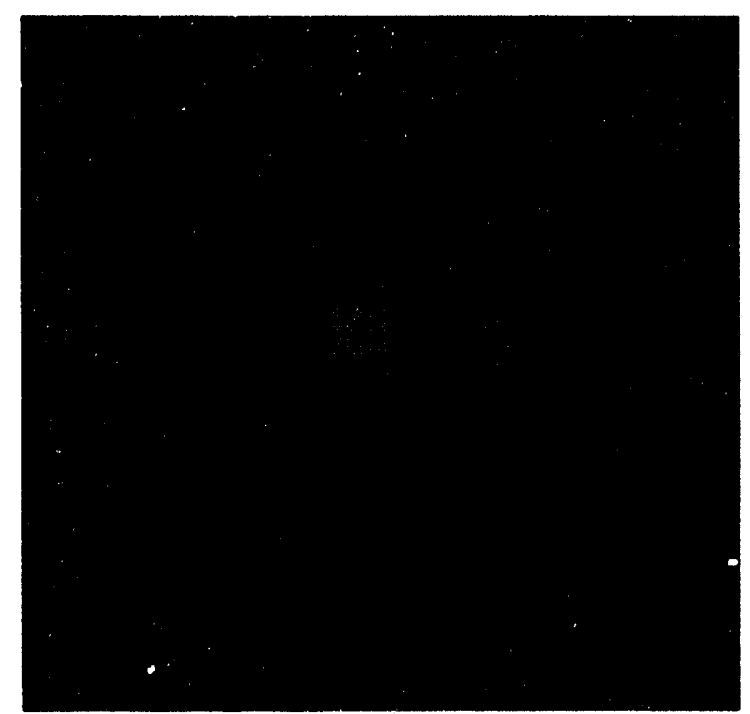

(a)

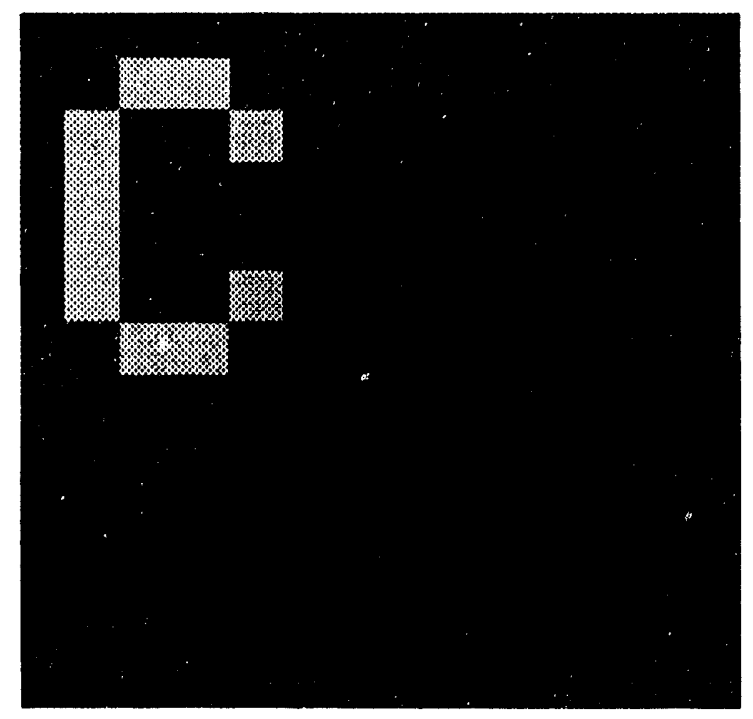

(b)

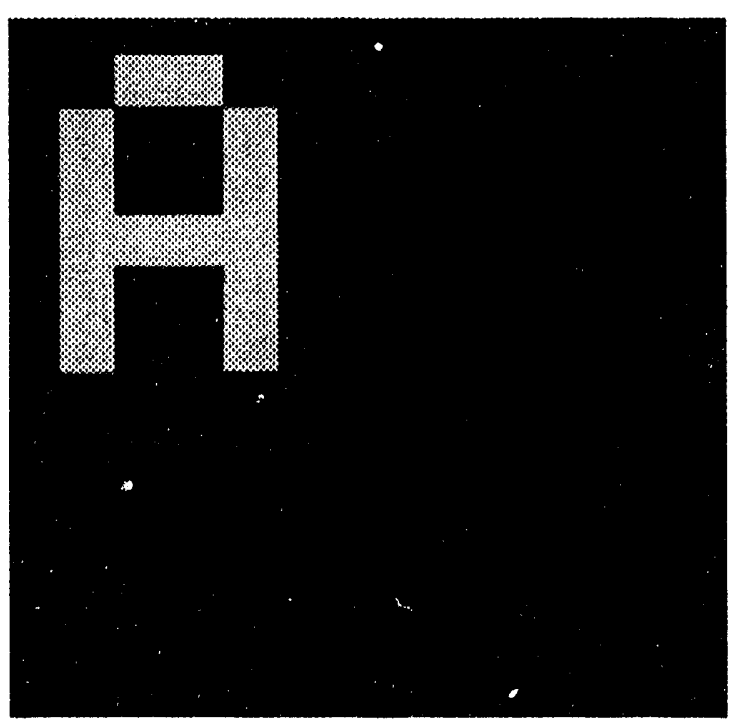

(c)

Figure 6. Blind deconvolution example. (a) Magnitude of a small ( $16 \times 16$ pixel) multiply-convolved complex image. (b), (c) Magnitudes of deconvolved ( $8 \times 8$ pixel) complex-image components.

Figure 7(a) depicts another multiply-convolved image, and Figures 7(b)-(d) illustrate the three $8 \times 8$ deconvolved image components. The operational code is currently set up to accommodate up to eight convolution components but could be increased arbitrarily by redimensioning some arrays. There is no fundamental limitation on the number of factors. 


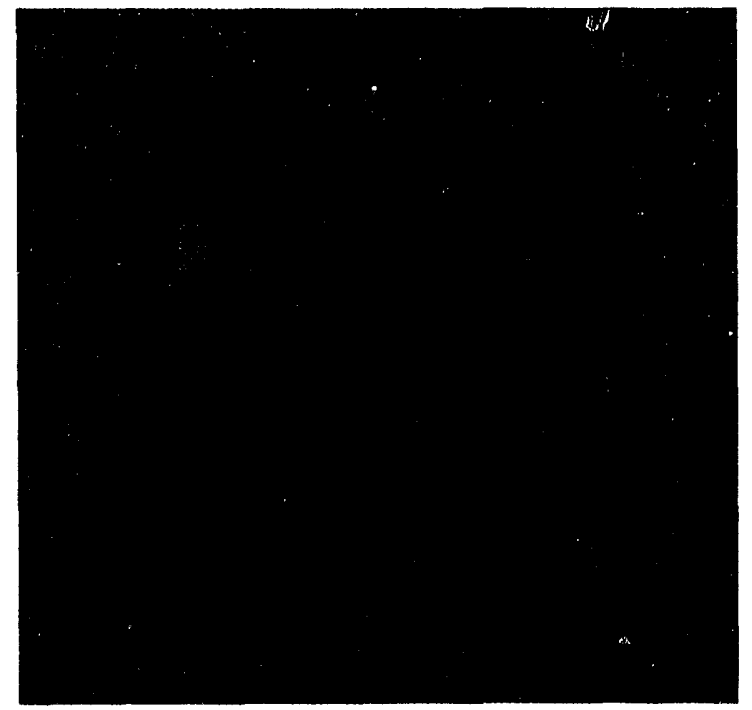

(a)

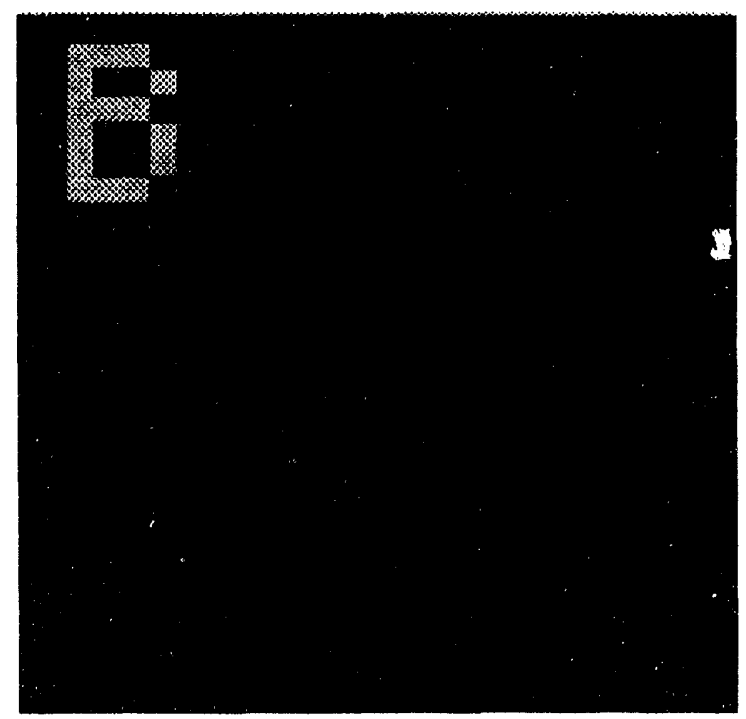

(c)

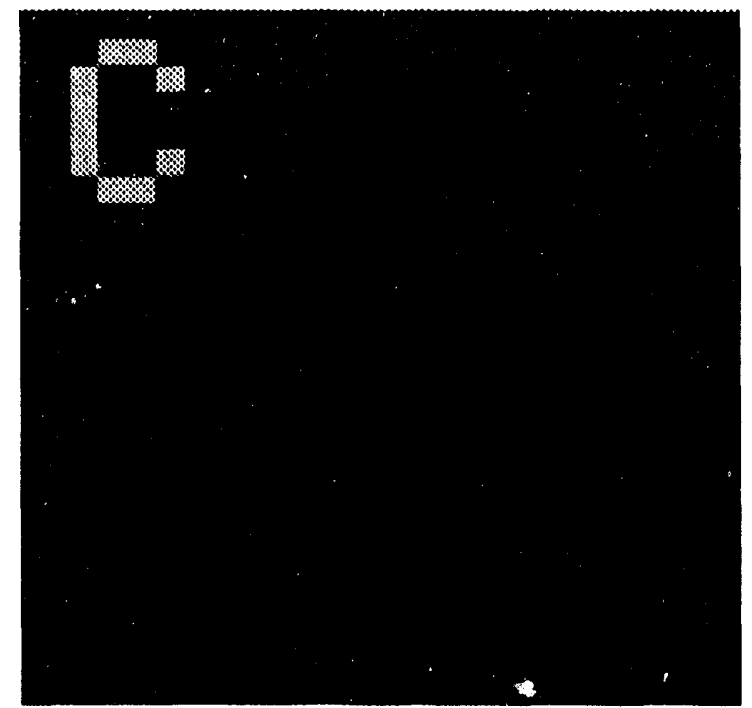

(b)

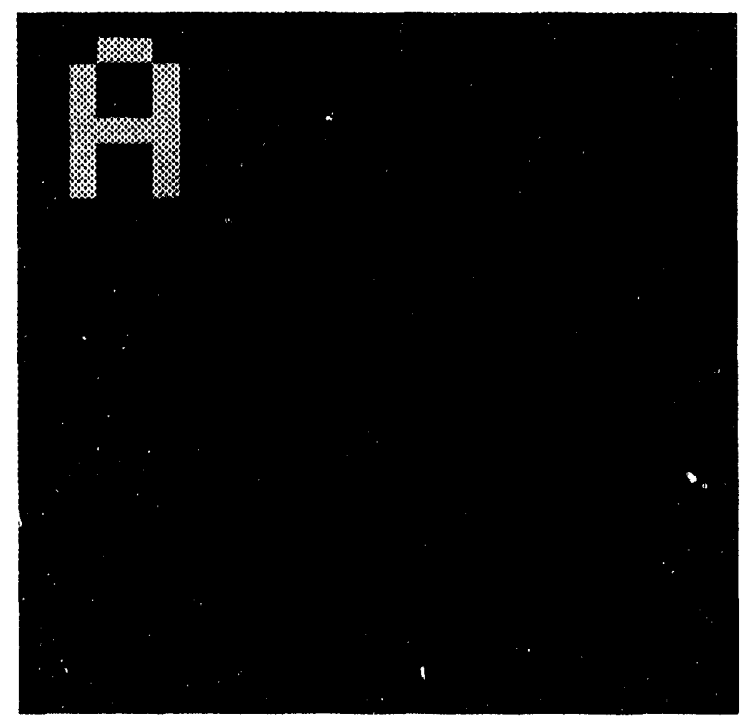

(d)

Figure 7. Multiple component convolution. (a) Magnitude of multiply-convolved complex image. (b)-(d) Magnitude of $8 \times 8$ pixel deconvolved complex-image components. 
Two final examples of the largest problem performed to date on a Sparc 2 work station (solution time was $\sim 70$ and $\sim 10$ hours, respectively) are shown in Figures 8 and 9. Figure 8(a) shows the convolution magnitude, and Figures $8(b)-(c)$ show the two $32 \times 32$ deconvolved images. Figure 9 (a) shows the magnitude of a $32 \times 32$ complex image [the phase was actually that shown in Figure 8(b)] convolved with a realvalued $8 \times 8$ point-spread function. Figure $9(\mathrm{~b})$ shows the convolution of the same 32 $\times 32$ image (now with constant phase making it essentially real valued) with the same $8 \times 8$ point-spread function. Figures $9(\mathrm{c})$ and $9(\mathrm{~d})$ show the $32 \times 32$ deconvolved image [complex in Figure 9(a), real-valued in Figure $9(\mathrm{~b})$ ] and the $8 \times 8$ point-spread function, respectively. The deconvolved images and point-spread function have the proper region of support and are upper-left justified in an array that has dimensions equal to or larger than the power-of-2 size necessary to hold the input data.

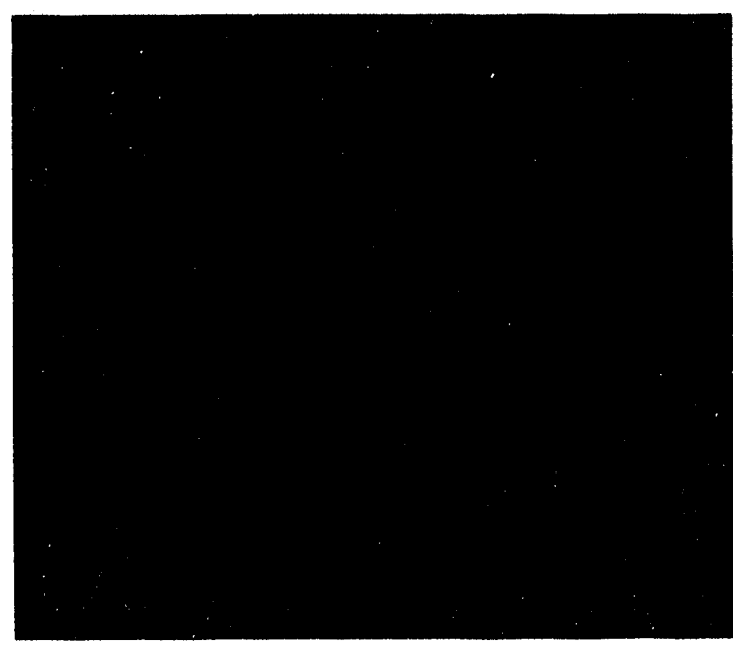

(a)

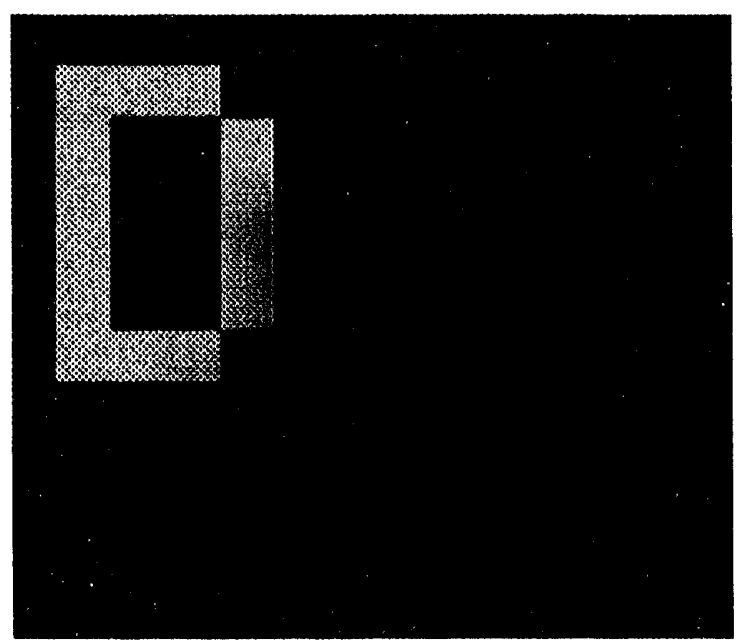

(b)

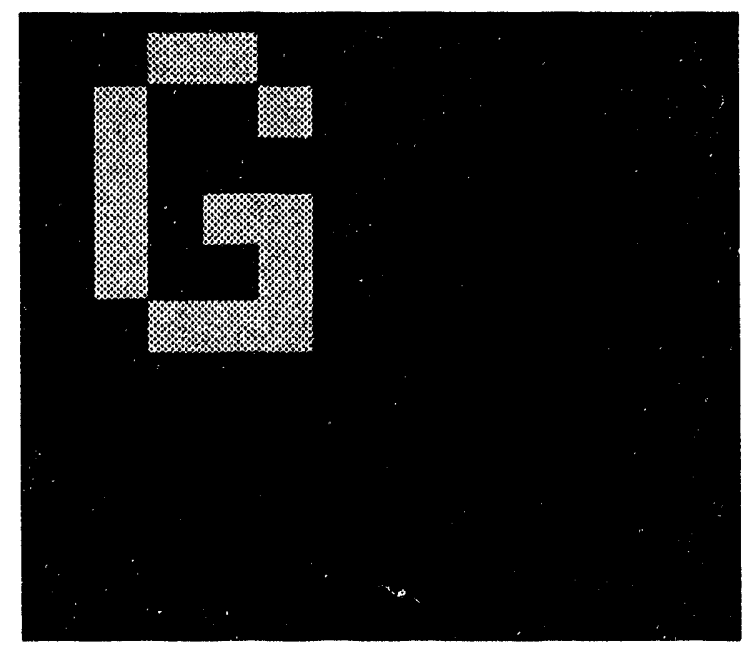

(c)

Flgure 8. Multiple component convolution. (a) $64 \times 64$ pixel magnitude of multiplyconvolved complex image. (b), (c) Magnitudes of $32 \times 32$ pixel deconvolved compleximage components. 


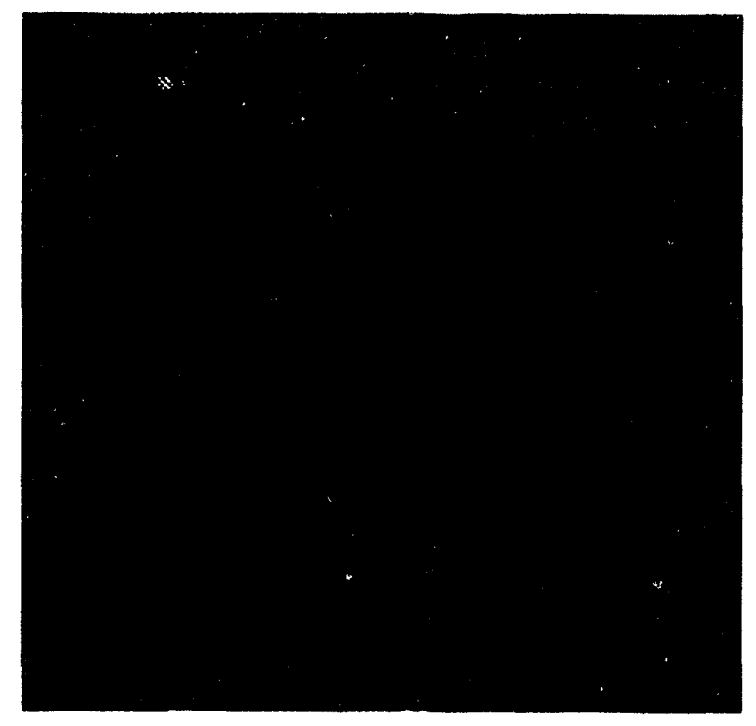

(a)

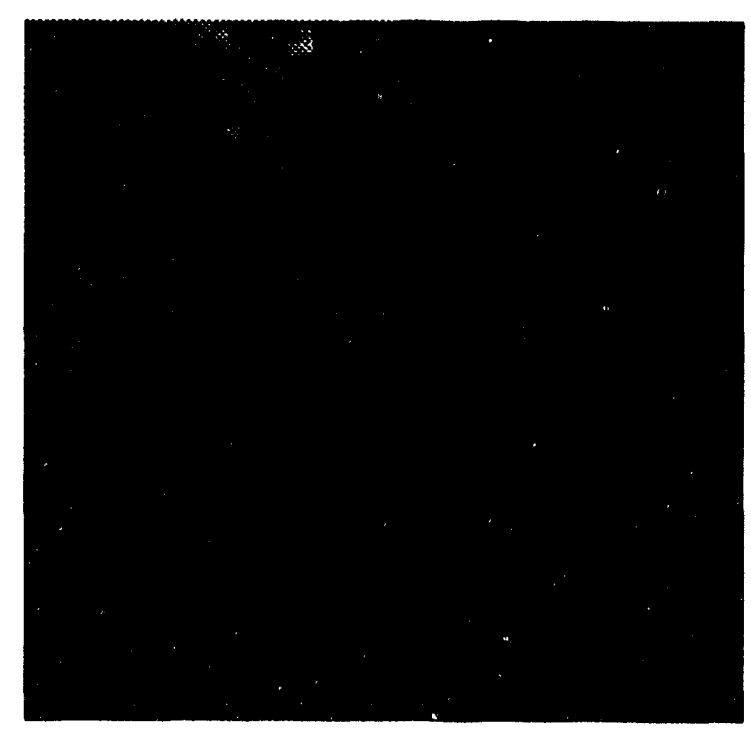

(c)

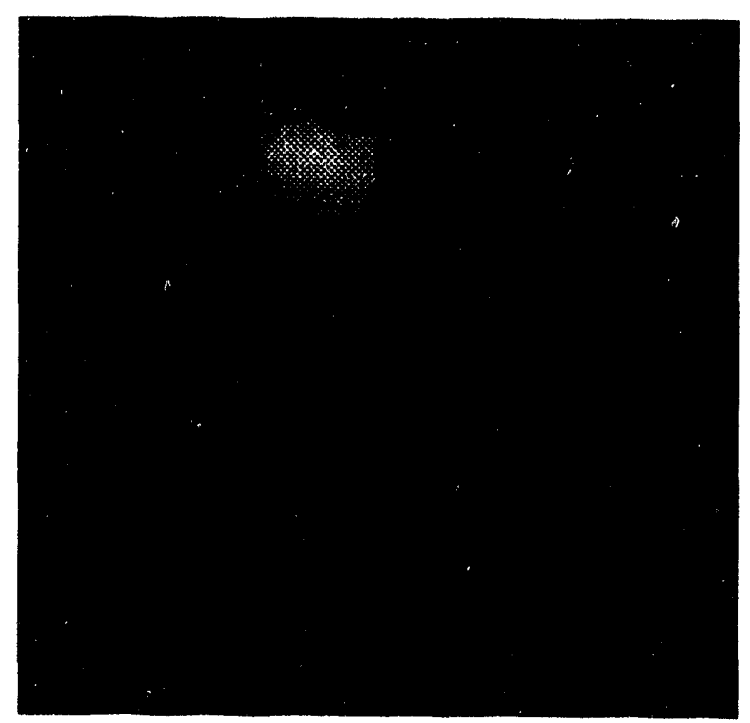

(b)

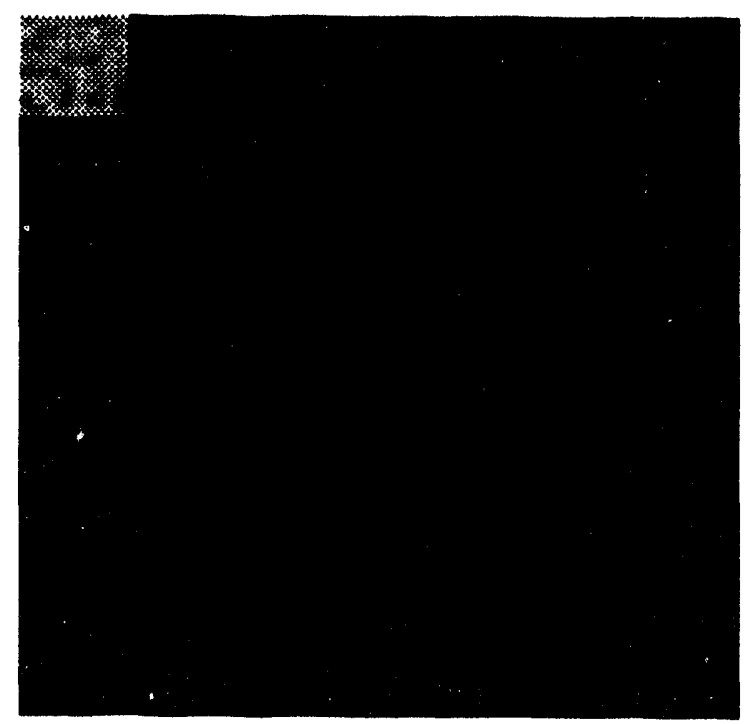

(d)

Flgure 9. Multiple component convolution. (a) Magnitude of a $32 \times 32$ complex image convolved with an $8 \times 8$ real-valued point-spread function. (b) Magnitude of a $32 \times 32$ real-valued image convolved with same $8 \times 8$ point-spread function. (c) Magnitude of a $32 \times 32$ deconvolved complex-image component [real-valued in 9(b)]. (d) Magnitude of a deconvolved $8 \times 8$ point-spread function. 


\section{Summary and Conclusion}

Although we have done our best, thus far, to incorporate many numerical tricks to ensure robustness, the computations required in root association and root tracking are quite complex and involved. During code development, as we systematically increased data set size, new difficulties showed up. We expect that as we try to approach realistic-sized imagery, other numerical difficulties may have to be addressed and solved if this approach is to be sound.

We have not yet dealt specifically with noise, except for that resulting from numerical rounding errors. All the numerical methods incorporated thus far were necessary to avoid problems with computation. We must, of course, explore the consequences of noise and further develop mitigating techniques. For example, we plan to investigate some graph-theoretical methods that operate on the association matrix. Graph-theoretical techniques have the potential to correct errors in the association matrix and/or yield the best factor separation when noise is encountered. As far as we know, this approach in dealing with noise has never been attempted or even proposed. In addition, we will always be searching for modifications to reduce the $O\left(N^{4}\right)$ computational burden.

This research was supported by the U. S. Department of Energy under contract number DE-AC04-76DP00789.

\section{References}

1. R. G. Lane and R. H. T. Bates, "Automatic multidimensional deconvolution," J. Opt. Soc. Am. A 4, 180-188 (1987).

2. B. L. K. Davey, R. G. Lane, and R. H. T. Bates, "Blind deconvolution of noisy complexvalued image," Opt. Comm. 69, 353-356 (1989).

3. R. H. T. Bates, B. K. Quek, and C. R. Parker, "Some implications of zero sheets for blind deconvolution and phase retrieval," J. Opt. Soc. Am. A 7, 468-479 (1990).

4. D. Izraelevitz and J. S. Lim, "A new direct algorithm for image reconstruction from Fourier transform magnitude, IEEE Trans. Acoust. Speech Signal Process. ASSP-35, 511519 (1987).

5. R. G. Lane, W. R. Fright, and R. H. T. Bates, "Direct phase retrieval," IEEE Trans. Acoust. Speech Signal Process. ASSP-35, 520-526 (1987).

6. N. E. Hurt, Phase Retrieval and Zero Crossings (Kluwer, Dordrecht, The Netherlands, 1989).

7. J. W. Goodman, International Trends in Optics (Academic, Boston, Mass., 1991).

8. H. Stark, Image Recovery, Theory and Applications (Academic, Orlando, Fla., 1987).

9. W. H. Press, B. P. Flannery, S. A. Teukolsky, and W. T. Vetterling, Numerical Recipes, The Art of Scientific Computing, Cambridge U. Press, Cambridge, 1986). 


\section{DISTRIBUTION:}

1 MS0321 E. H. Barsis, 1400

1 MS1111 S. J. Dosanjh, 1402

1 MS0318 G. S. Davidson, 1403

1 MS0821 J. A. Ang, 1404

1 MS1111 W. J. Camp, 1421

1 MS1110 R. C. Allen, 1422

1 MS1110 E. F. Brickell, 1423

6 MS1110 D. C. Ghiglia, 1423

1 MS1109 A. L. Hale, 1424

1 MS0441 J. H. Biffle, 1425

1 MS0819 J. M. McGlaun, 1431

1 MS0820 P. Yarrington, 1432

1 MS0821 P. L. Stanton, 1433

1 MS0439 D. R. Martinez, 1434

1 MS9018 Central Technical Files, 8523-2

5 MS0899 Technical Library, 7141

1 MS0619 Technical Publications, 7151

10 MS0100 Document Processing for DOE/OSTI, 7613-2 

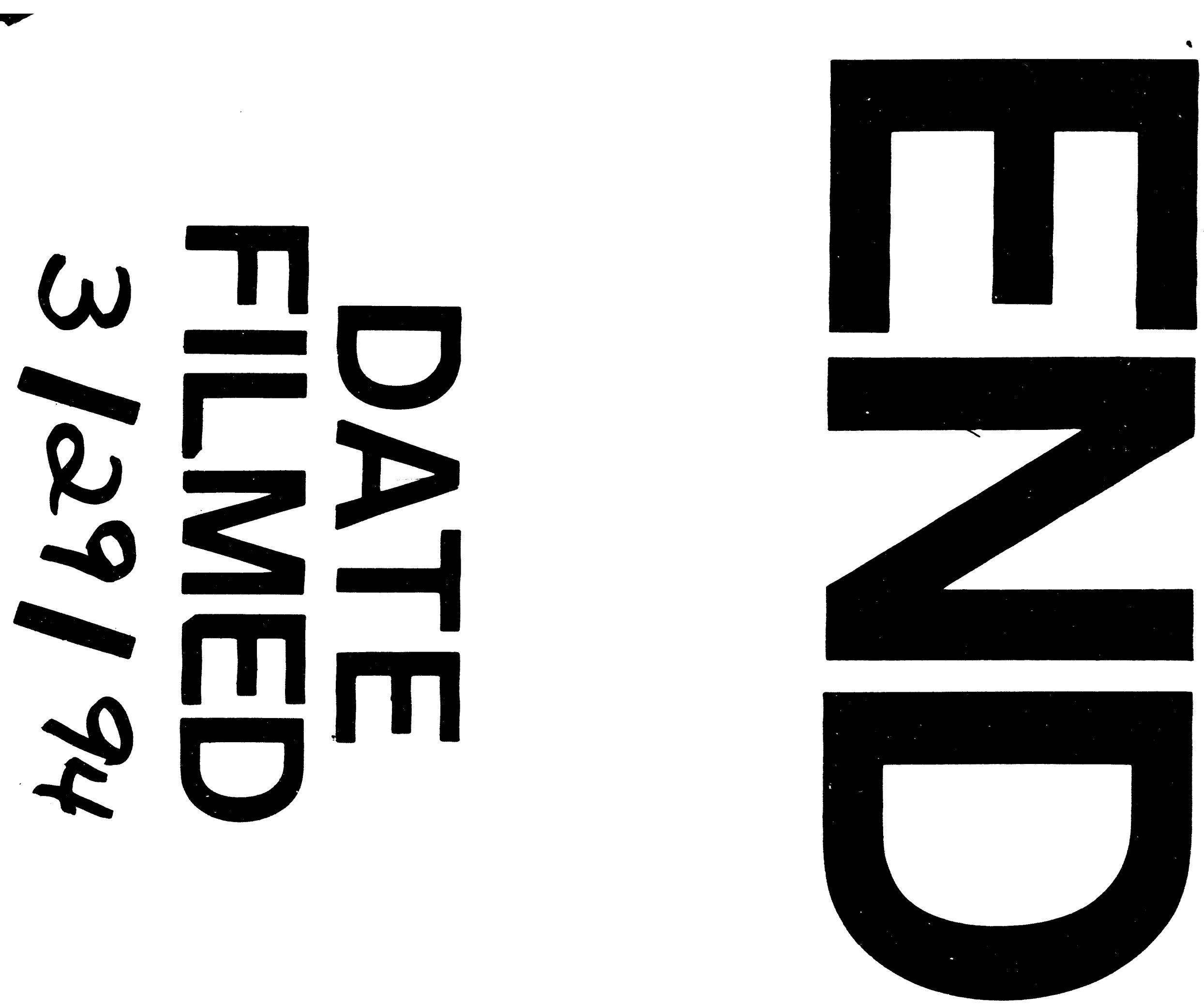

ג 
